\title{
WEAK CAT-OPERADS
}

\author{
KOSTA DOŠEN AND ZORAN PETRIĆ
}

Mathematical Institute, SANU, Knez Mihailova 36, p.f. 367, 11001 Belgrade, Serbia

e-mail address: \{kosta,zpetric\}@mi.sanu.ac.rs

\begin{abstract}
An operad (this paper deals with non-symmetric operads) may be conceived as a partial algebra with a family of insertion operations, which correspond to substitution of an operation within an operation. These insertion operations are Gerstenhaber's circle-i products, and they satisfy two kinds of associativity, one of them involving commutativity. A Cat-operad is an operad enriched over the category Cat of small categories, as a 2category with small hom-categories is a category enriched over Cat. This means that the operadic operations of the same arity in a Cat-operad do not make just a set, but they are the objects of a small category. The notion of weak Cat-operad is to the notion of Cat-operad what the notion of bicategory is to the notion of 2-category. This means that the equations of operads like associativity of insertions are replaced by isomorphisms in a category. The goal of this paper is to formulate conditions concerning these isomorphisms that ensure coherence, in the sense that all diagrams of canonical arrows commute. This is the sense in which the notions of monoidal category and bicategory are coherent. (The coherence of monoidal categories, which is due to Mac Lane, is the best known coherence result.) The coherence proof in the paper is much simplified by indexing the insertion operations in a context-independent way, and not in the usual manner. This proof, which is in the style of term rewriting, involves an argument with normal forms that generalizes what is established with the completeness proof for the standard presentation of symmetric groups. This generalization may be of an independent interest, and related to other matters than those studied in this paper. Some of the coherence conditions for weak Cat-operads lead to the hemiassociahedron, which is a polyhedron related to, but different from, the three-dimensional associahedron and permutohedron.
\end{abstract}

2012 ACM CCS: [Theory of computation]: Logic-Proof theory; [Mathematics of computing]: Discrete mathematics - Combinatorics - Combinatorics on words; Discrete mathematics - Graph theoryHypergraphs.

2010 Mathematics Subject Classification: 03B22, 03G30, 08A55, 18A15, 18D05, 18D20, 18D50, 52B10, $55 \mathrm{P} 48$.

Key words and phrases: operad, non-symmetric operad, Cat-operad, weak Cat-operad, 2-category, bicategory, multicategory, nominal arity, coherence, symmetric groups, hemiassociahedron, associahedron, permutohedron.

니
DOI:10.2168/LMCS-11(1:10)2015

K. Došen and Z. Petrić

(c) Creative Commons 


\section{Contents}

\section{Introduction}

PART I

2. The operad $\mathcal{O}$

3. The structure $\mathcal{O}_{e}$

4. $\mathcal{O}$ and $\mathcal{O}_{e}$

5. The structure $\mathcal{O}_{u}$

6. $\mathcal{O}_{e}$ and $\mathcal{O}_{u}$

PART II

7. The category $\mathcal{W O}_{u}^{-}$

8. The category $\mathcal{W O}_{u}$

9. The category $\mathcal{W O}_{e}^{-}$

10. The category $\mathcal{W O}_{e}$

11. $\mathcal{W O}_{e}$ and $\mathcal{W O}_{u}$

12. Operads, Cat-operads and weak Cat-operads

13. $\mathcal{W O}_{e}^{-}$and hemiassociahedra

\section{PART III}

14. Coherence of $\mathrm{Mon}_{u}$

15. The category $\mathcal{W O}_{u}^{\theta}$

16. $\mathcal{C}_{\Gamma}$ and $\mathcal{B C}_{\Gamma}$

17. Coherence of $\mathcal{C}_{\Gamma}$

18. $\mathcal{C}_{\Gamma}$ and $\mathcal{W} \mathcal{O}_{u}^{\theta}$ - Coherence of $\mathcal{W} \mathcal{O}_{e}$

References

\section{INTRODUCTION}

An operad may be conceived as a partial algebra whose elements, called operadic operations, are of various arities; to these elements as arguments one applies partial binary operations — these are operations applied to operadic operations, partiality being induced by arity which we call insertions. We have this new name to distinguish insertions from the related partial operation of composition in categories, which will appear together with insertions later in this paper. Insertions correspond to Gerstenhaber's " ${ }_{i}$-products" (see [MSS02], Sections I.1.3 and II.1.3, and the "circle- $i$ " of [Lei04], Section 2.3) or to Gentzen's cut (see Gen35).

For insertions one assumes two kinds of associativity, one of them involving commutativity up to a certain point (see the equations (assoc 1) and (assoc 2) in Section 2 below, and related equations given later). One assumes also a unit operadic operation and appropriate equations tying it to insertions. When this unit is missing we have a non-unitary operad; otherwise, the operad is unitary (for this terminology see [MSS02], Section II.1.3). This notion of operad, with which we deal in this paper, is not the original symmetric notion, but the non-symmetric (non- $\Sigma$ ) notion (see MSS02, Section I.1.3, May72 and Lei04).

A Cat-operad is an operad enriched in the category Cat of all small categories, whose arrows are functors, as a 2-category is a category enriched over Cat (provided the homcategories of the 2-category are small; see [MLa98, Section XII.3). The operadic operations 
of the same arity in a Cat-operad do not make just a set, but they are the objects of a small category, and the structure of the operad involving insertions is related by some assumptions to the categorial structure (see the precise definition in Section 12).

The notion of weak Cat-operad will be to the notion of Cat-operad what the notion of bicategory is to the notion of 2-category (see [MLa98, Section XII.6). The equations of operads like associativity of insertion are replaced by isomorphisms in the categorial structure, and one has to make assumptions concerning these isomorphisms to ensure coherence. Coherence means here, as in Mac Lane's original use of the term, that "all diagrams commute", i.e. all diagrams of canonical arrows do so. Coherence for weak Cat-operads is like Mac Lane's coherence for monoidal categories (see [MLa63] and [MLa98], Section VII.2), and like coherence for bicategories of [MLP85].

Besides this motivation from the theory of operads, this paper may be taken as being motivated by the theory of multicategories. Multicategories, as conceived by Lambek in [Lam69] and [Lam89], are a generalization of the notion of operad with insertions primitive, where not only the arity of the operadic operations counts. Instead of one-sorted operadic operations we have many-sorted operations (see also Section 12). This notion is the categorial reconstruction of Gentzen's sequents of Gen35] (singular sequents, with a single conclusion), and it is interesting for proof theory.

For multicategories we have, as for operads with insertions primitive, two kinds of associativity (the corresponding equations analogous to (assoc 1) and (assoc 2) are in Lambek's papers), and the mathematics involved in finding a weak notion of multicategory analogous to our weak Cat-operads would not differ essentially from what we have in this paper. That would only involve additions not influencing in a significant way the mathematical core. Our category $\mathcal{W O}_{u}$ in Part II is not far from this notion, but in this paper we will not go further into this matter.

Yet another motivation for the present paper would come from matters investigated in [DP06]. There one finds insertions as operations used to present in a non-standard manner an algebra with a free binary operation (a groupoid in the sense of universal algebra), with or without unit. These insertions satisfy again the two kinds of associativity. As, by replacing the equations by isomorphisms, the two kinds of associativity are weakened in the notion of weak Cat-operad, so this can be done in the context of [DP06]. In this weakened context we would be interested in the constructing of an element of our algebra rather than in this element itself, which is related to matters treated in [DP10] and in Section 13 of this paper.

A motivation for this paper is also in the theory of polyhedra related to permutohedra and associahedra (see below and Section 13). This matter is also interesting because, due to the convexity of the polyhedra in question (which follows from the realizations presented in [DP11]), it would yield an alternative proof of coherence in the style of Stasheff, as a proof of Mac Lane's coherence for monoidal categories may be based on [Sta63] (see Kap93 and [DP07]).

Up to now we have been concerned with the motivation for our paper. We will now survey its content. Our goal is to formulate a notion of weak Cat-operad, spell out the coherence conditions, and demonstrate coherence. The paper is organized in three parts.

In the first part (Sections 2-6) we introduce the free unitary operad $\mathcal{O}$ obtained from a generating set $G$ of free operadic operations. The general notion of operad with insertions primitive is based on $\mathcal{O}$ (see Section 12). Next we formulate a partial algebraic structure we call $\mathcal{O}_{e}$, which is essentially a notational variant of $\mathcal{O}$. The structure of $\mathcal{O}_{e}$ is less handy for 
basing the general notion of operad on it; for that $\mathcal{O}$ is better. The structure $\mathcal{O}_{e}$ is however handier to work with than $\mathcal{O}$ when it comes to indexing the insertions.

Instead of indexing insertions by natural numbers, which stand for the number of the place where the insertion is made counting from the left, we introduce a name for that place, which is a word in the alphabet of natural numbers, a finite sequence of natural numbers. While the number of the place is context-dependent - it may increase when further insertions are made on its left - the name we introduce is context-independent; it is invariant. The arity of an operadic operation, the ordinary numerical arity, which is just a natural number in $\mathcal{O}$ and in the general notion of operad, becomes a set of our names. We call such a set a nominal arity. The nominal arities of this paper may be conceived as made of leaves of trees (see the examples in Section 13).

Invariance makes $\mathcal{O}_{e}$ handier in the following sense. At the cost of making more involved the indexing of insertions, we have simplified the equations expressing the two kinds of associativity.

This will become very important when these equations are replaced by isomorphisms in the second part of the paper (Sections 7-13), and in the remainder. Expressing the coherence conditions for the isomorphisms using numerical arities, instead of our nominal arities, is possible, but it would be extremely and unnecessarily complicated (for an example see Section 12).

We introduce also in the first part a structure more general than $\mathcal{O}_{e}$, which we call $\mathcal{O}_{u}$. The structure $\mathcal{O}_{e}$ corresponds, roughly, to just one level of $\mathcal{O}_{u}$, which may be understood as a multicategory freely generated by some particular generators (see the end of Section 5).

In $\mathcal{O}_{u}$ we have instead of a family of insertions, indexed by our names, just one partial operation of insertion. This is achieved at the cost of, so to speak, moving the indices of insertions into the structure in order to distinguish various occurrences of the same element of the structure, various occurrences of the same operadic operation.

Because of this diversification the structure may look more complicated. As a matter of fact, it is still handier to work with. The advantage of having just one insertion operation proves very big when it comes to axiomatizing the coherence conditions, in the second part of the paper.

With $\mathcal{O}_{u}$ the two kinds of associativity become, on the one hand, just plain associativity and, on the other hand, associativity mixed with commutativity, of a single insertion operation. The coherence conditions for the corresponding isomorphisms reproduce now to a great extent the coherence conditions for associativity and commutativity, well known from Mac Lane's coherence results for monoidal and symmetric monoidal categories (see MLa63 and MLa98, Chapter VII), and from the standard presentation of symmetric groups. There are however new coherence conditions mixing the two kinds of associativity isomorphisms; let us call them mixed coherence conditions.

Proving that the previously known coherence conditions together with the new mixed coherence conditions are sufficient for coherence is possible to do with a single insertion operation, and we do that in the third, and last, part of the paper (Sections 14-18). We suppose it would be possible to prove that also directly in the $\mathcal{O}_{e}$ version, with a family of insertions, but it would be much more complicated, and moreover it would be unnecessarily so. The complications would not alter the underlying combinatorial core - they would just obscure it.

In the second part of the paper we axiomatize our coherence conditions in the $\mathcal{O}_{u}$ and $\mathcal{O}_{e}$ versions, which results in the categories $\mathcal{W O}_{u}$ and $\mathcal{W} \mathcal{O}_{e}$. We establish that $\mathcal{W} \mathcal{O}_{u}$ is the 
disjoint union of isomorphic copies of $\mathcal{W} \mathcal{O}_{e}$, and we use $\mathcal{W} \mathcal{O}_{e}$ to give our notion of weak Cat-operad in Section 12.

The last section of that part, Section 13, is optional, and a reader not interested in the matters treated there may skip it, and continue reading the remainder. Through this section our paper is however connected with important and interesting matters of algebraic topology and combinatorics. It provides an insight into the regularity underlying the equations with which we define weak Cat-operads. In Section 13 we consider how the mixed coherence conditions engender a new kind of polyhedron, related to, but different from, the three-dimensional associahedron and permutohedron. The family to which this polyhedron, called hemiassociahedron, belongs (the family includes also the three-dimensional cyclohedron) is investigated in [DP10] and [DP11] (which should be consulted for further references). The mixed conditions may also however lead in some cases to the three-dimensional associahedron and permutohedron, which provides another perspective on these well-known polyhedra tied to associativity and commutativity. The hemiassociahedron arises in the non-unitary structure of our weak Cat-operads, as the associahedron and permutohedron arise in the non-unitary structure of monoidal categories and symmetric groups.

In the third part of the paper we prove the sufficiency of our conditions for coherence (these conditions are of course also necessary). We do that with $\mathcal{W} \mathcal{O}_{u}$, which, as we said, is easier to work with. We strictify first the monoidal structure of $\mathcal{W} \mathcal{O}_{u}$ (all the arrows of that structure become identity arrows), which leaves a category $\mathcal{W} \mathcal{O}_{u}^{\theta}$, equivalent with $\mathcal{W} \mathcal{O}_{u}$, which is similar to a symmetric strictly monoidal category.

To prove that $\mathcal{W} \mathcal{O}_{u}^{\theta}$ is a preorder (i.e. that there is not more than one arrow in it with a given source and target), which is what coherence here amounts to, we reconsider the standard presentation of symmetric groups, and a proof that this presentation is complete. This proof, which involves a reduction to a normal form, implicit already in Moore's paper [Mo896] (the first paper to deal with the matter), yields, as a matter of fact, something more, pertaining to a wider family of structures.

In this family we have categories we call $\mathcal{C}_{\Gamma}$, which generalize symmetric strictly monoidal categories; symmetric groups belong to the family as one-object categories. All one has in the categories $\mathcal{C}_{\Gamma}$ comes from the symmetric structure, but this structure may be incomplete; it may, roughly speaking, have gaps. It need not even give a groupoid in the categorial sense (its arrows need not be invertible, they need not be isomorphisms). The category $\mathcal{W O}_{u}^{\theta}$ is just a particular $\mathcal{C}_{\Gamma}$, and that it is a preorder follows from a coherence result for an arbitrary $\mathcal{C}_{\Gamma}$. This coherence involves graphs corresponding to permutations, but in connection with $\mathcal{W} \mathcal{O}_{u}^{\theta}$, because of diversification (see above), the graphs need not be mentioned. It follows that $\mathcal{W} \mathcal{O}_{u}$ is a preorder, which implies that $\mathcal{W} \mathcal{O}_{e}$ is a preorder, and with that our notion of weak Cat-operad is justified. (For other examples that fall within the range of this general coherence result one may consult [DP06a and DP12].)

Throughout the paper we distinguish matters pertaining to unitary operads from those pertaining to non-unitary operads. The non-unitary versions of our operads, categories and related structures have a superscript ${ }^{-}$in their names. Whatever we established for the unitary versions can be established for the non-unitary ones, which make the non-unitary core of our notions. As a matter of fact, the more interesting mathematics pertains to the non-unitary notions (as Section 13 illustrates).

We do not claim that our notion of weak Cat-operad is the right notion of weak Catoperad in general, but that it is the right notion if we are motivated by coherence involving 
insertions. In our definition, this coherence has been combinatorially analyzed by our axiomatic equations. We prove that no equation is missing.

The notion of monoidal category was introduced in a non-axiomatic way via coherence by Bénabou in Bén63, and in the equational axiomatic way, such as we favour, by Mac Lane in MLa63. Mac Lane's definition is the standard one, while Bénabou's is rarely mentioned. For Bénabou, coherence is built into the definition, and for Mac Lane it is a theorem. One could analogously define the theorems of classical propositional logic as being the tautologies (this is done, for example, in [CK73], Sections 1.2-3), in which case completeness would not be a theorem, but would be built into the definition. Analogously to what was done for monoidal categories in [MLa63, and later for bicategories in [MLP85, we are not only proposing a definition, but we are proving a coherence theorem concerning it.

An anonymous referee of our text asks whether our notion of weak Cat-operad is equivalent with the notion of pseudo-operad of [DS01. Another anonymous referee is convinced that the right notion of weak Cat-operad is given by, as suggested to him by Mark Weber, "pseudo-algebras of a monad for which the strict algebras are the existing notion of Catoperad" (without going into details), and that referee wonders whether this notion is the "same" as a ours.

To start answering these questions one would have to consider first the difference in language, since the other notions are not based like ours on insertions. If the other notions could be based on insertions (in the non-unitary case defining insertions would pose a problem), the question would reduce to the question whether these other notions of weak Cat-operad require coherence (in our and Mac Lane's sense) involving insertions, which seems possible. If they require this coherence, then our paper provides a positive answer to the question. It would show that notions, which are not like Mac Lane's, are equivalent with a notion in the style of Mac Lane.

The study of this matter - whether the alternative notions could be based on insertions, and whether they require coherence involving insertions - would be a study of these alternative notions, conducted independently of the content of this paper. This is a different topic, best left for a separate treatment, because, as far as we can see, to be presented with sufficient detail this would require a lot of space.

PART I

\section{THE OPERAD $\mathcal{O}$}

Let $G$ be a set, for whose members we use $x, y, z, \ldots$, perhaps with indices, and let $\alpha_{G}: G \rightarrow \mathbf{N}$ be a function; $\alpha_{G}(x)$ is intuitively the arity of $x$. The elements of $G$ are the free, generating, primitive operadic operations of the operad $\mathcal{O}$, which is the unitary operad freely generated out of $G$ and $\alpha_{G}$ in the following manner. (As a matter of fact, mentioning $\alpha_{G}: G \rightarrow \mathbf{N}$ is enough; it carries the information about $G$.)

We define first inductively the set of terms of $\mathcal{O}$; these terms will stand for the operadic operations of $\mathcal{O}$. Together with the terms of $\mathcal{O}$ we define simultaneously a function $\alpha$ from the terms of $\mathcal{O}$ to $\mathbf{N}$; the values of this function are intuitively the arity. Here are the three clauses of these two simultaneous inductive definitions:

(0) if $x \in G$, then $x$ is a term; $\quad \alpha(x)=\alpha_{G}(x)$;

(1) $\quad \mathbf{I}$ is a term; $\alpha(\mathbf{I})=1$; 
(2) if $\varphi$ and $\gamma$ are terms and $1 \leq n \leq \alpha(\gamma)$, then $\gamma \triangleleft_{n} \varphi$ is a term;

$\alpha\left(\gamma \triangleleft_{n} \varphi\right)=\alpha(\gamma)-1+\alpha(\varphi)$.

Officially, in (2) we should have $\left(\gamma \triangleleft_{n} \varphi\right)$ instead of $\gamma \triangleleft_{n} \varphi$, but, as usual, the outermost parentheses of these and other terms later will be taken for granted, and omitted. We use $\varphi, \gamma, \chi, \ldots$, perhaps with indices, for the terms of $\mathcal{O}$.

The term I stands for the unit operadic operation, and $\triangleleft_{k}$ stands for a partial operation of insertion. The expression $\gamma \triangleleft_{n} \varphi$ does not become a term for every substitution for $n, \varphi$ and $\gamma$; substitutions for $n$ and $\gamma$ are restricted. We express this by saying that $\gamma \triangleleft_{n} \varphi$ is legitimate when $1 \leq n \leq \alpha(\gamma)$.

Analogously, substitutions will be restricted for the equations between terms of the operad $\mathcal{O}$. As for terms, we express this by saying that $\varphi=\gamma$ is legitimate when $\alpha(\varphi)=\alpha(\gamma)$. An equation cannot hold between terms of different arity.

The equations of $\mathcal{O}$ between terms are given through an axiomatic equational system, and the operadic operations of $\mathcal{O}$ will be formally equivalence classes of terms of $\mathcal{O}$ such that these equations are satisfied. Besides $\varphi=\varphi$, the axiomatic equations of $\mathcal{O}$ are the following:

(unit) $\quad \varphi \triangleleft_{n} \mathbf{I}=\varphi, \quad \mathbf{I} \triangleleft_{1} \varphi=\varphi$,

(assoc 1) $\left(\chi \triangleleft_{n} \gamma\right) \triangleleft_{m} \varphi=\chi \triangleleft_{n}\left(\gamma \triangleleft_{m-n+1} \varphi\right), \quad$ provided $n \leq m<n+\alpha(\gamma)$,

(assoc 2) $\left(\chi \triangleleft_{n} \gamma\right) \triangleleft_{m} \varphi=\left(\chi \triangleleft_{m-\alpha(\gamma)+1} \varphi\right) \triangleleft_{n} \gamma$, provided $n+\alpha(\gamma) \leq m$.

The proviso for (assoc 1) may be derived from the legitimacy of $\gamma \triangleleft_{m-n+1} \varphi$, for which we must have $1 \leq m-n+1 \leq \alpha(\gamma)$. So, as a matter of fact, this proviso need not have been mentioned, and in the future we will not always mention such provisos, which may be inferred from the legitimacy of an equation, or of our notation for terms. We assume that all the expressions for terms that occur in an equation are legitimate.

The proviso for (assoc 2) is not derivable in this manner. This equation could be replaced by

$$
\left(\chi \triangleleft_{n} \gamma\right) \triangleleft_{m} \varphi=\left(\chi \triangleleft_{m} \varphi\right) \triangleleft_{n+\alpha}(\varphi)-1 \gamma, \quad \text { provided } m<n .
$$

The remaining equations of $\mathcal{O}$ are derived with the help of the rules of symmetry and transitivity of $=$, and of the rule of $\triangleleft_{n}$-congruence:

from $\varphi_{1}=\varphi_{2}$ and $\gamma_{1}=\gamma_{2}$ derive $\gamma_{1} \triangleleft_{n} \varphi_{1}=\gamma_{2} \triangleleft_{n} \varphi_{2}$,

provided both sides of the last equation are legitimate. As a matter of fact, it is superfluous to state this proviso; we understand rules like $\triangleleft_{n}$-congruence always with such provisos.

Once we have defined this axiomatic equational system, it can be verified by an easy induction on the length of derivation that for every equation $\varphi=\gamma$ of $\mathcal{O}$ we have $\alpha(\varphi)=$ $\alpha(\gamma)$. So these equations are indeed legitimate.

This concludes our definition of the operad $\mathcal{O}$. This operad may be conceived as a partial algebra, i.e. algebra with partial operations, $\left\langle C(\mathcal{O}),\left\{\triangleleft_{n} \mid n \in \mathbf{N}^{+}\right\}\right.$, I $\rangle$, with $C(\mathcal{O})$, the carrier of $\mathcal{O}$, being the set of operadic operations of $\mathcal{O}$.

The non-unitary operad $\mathcal{O}^{-}$freely generated out of $G$ and $\alpha_{G}$ is defined like $\mathcal{O}$ save that we omit clause (1) from the definition of terms, and we omit (unit) from the axiomatic equations.

\section{The Structure $\mathcal{O}_{e}$}

Before we introduce $\mathcal{O}_{e}$ we deal with preliminary matters concerning nominal arities. 
An $\mathbf{N}^{+}$-word is a finite (possibly empty) sequence of natural numbers greater than 0 . We use $a, b, c, \ldots$, perhaps with indices, for $\mathbf{N}^{+}$-words; we reserve $e$ to denote the empty $\mathbf{N}^{+}$-word.

An $\mathbf{N}^{+}$-language is a set of $\mathbf{N}^{+}$-words. An $\mathbf{N}^{+}$-language is called a nominal arity when there are no two distinct $\mathbf{N}^{+}$-words of the form $a$ and $a b$ in it; i.e. such that one is a proper initial segment of the other. This definition allows also infinite nominal arities, but in this paper we have use only for finite ones. The empty $\mathbf{N}^{+}$-language and every singleton $\mathbf{N}^{+}$-language are nominal arities. We use $X, Y, Z, \ldots$, perhaps with indices, for nominal arities.

We say that the $\mathbf{N}^{+}$-word $a$ is a prefix of the nominal arity $X$ when for every $c$ in $X$ we have that $c$ is of the form $a b$; i.e., $a$ is an initial segment, not necessarily proper, of every member of $X$. Note that $a$ may be $e$, which is a prefix of every nominal arity. Note also that $X$ may have more than one prefix, of which $e$ is always one. It is trivially satisfied that every $a$ is a prefix of the empty nominal arity. The set of prefixes of $X$ is denoted by $P_{X}$.

For every $\mathbf{N}^{+}$-language $M$, and, in particular, for $M$ a nominal arity, let

$$
a \cdot M=_{d f}\{a b \mid b \in M\} .
$$

We have, of course, $e \cdot M=M$.

Every nominal arity is linearly ordered by the lexicographical order $\prec$, whose definition for nominal arities is simpler, and is given by the following:

$$
a_{1} \prec a_{2} \quad \text { iff } \exists a, b, c\left(\exists n, m \in \mathbf{N}^{+}\right)\left(a_{1}=a n b \quad \& \quad a_{2}=a m c \quad \& n<m\right) .
$$

If $a \in P_{X} \cap Y$, then we define the result of inserting $X$ in $Y$ at $a$ :

$$
Y \triangleleft_{a} X=_{d f}(Y-\{a\}) \cup X .
$$

Note that this union is disjoint. Otherwise, for some $b$ in $Y$ different from $a$ we would have that it is in $X$, and hence that it has $a$ as an initial segment; this contradicts the assumption that $Y$ is a nominal arity. We conclude in a similar manner, by going through all possible cases, that $Y \triangleleft_{a} X$ is a nominal arity. The expression $Y \triangleleft_{a} X$ is legitimate when $a \in P_{X} \cap Y$. For $|M|$ being the cardinality of the set $M$, we have the following.

Remark 3.1. $|a \cdot X|=|X|, \quad\left|Y \triangleleft_{a} X\right|=|Y|-1+|X|$.

For the second equation we rely on the disjointness mentioned after the definition of $\triangleleft_{a}$. We also have the following.

Remark 3.2. $a \cdot P_{X} \subseteq P_{a \cdot X}, \quad P_{Y} \subseteq P_{Y \triangleleft_{a} X}$.

The inclusion converse to the first one holds only if $a$ is $e$; if $a$ is not $e$, then $e \in P_{a \cdot X}$ and $e \notin a \cdot P_{X}$. The inclusion converse to the second one does not hold for $Y=\{a\}$ and $X=\emptyset$. We also have the following two remarks.

Remark 3.3. $a \cdot(b \cdot X)=a b \cdot X, \quad a \cdot\left(Y \triangleleft_{b} X\right)=a \cdot Y \triangleleft_{a b} a \cdot X$.

Remark 3.4. For $a \in Y$ and $b \in Z,\left(Z \triangleleft_{b} Y\right) \triangleleft_{a} X=Z \triangleleft_{b}\left(Y \triangleleft_{a} X\right)$; for $a, b \in Z,\left(Z \triangleleft_{b} Y\right) \triangleleft_{a} X=\left(Z \triangleleft_{a} X\right) \triangleleft_{b} Y$.

The condition $b \in Z$ is implied by the legitimacy of $Z \triangleleft_{b} Y$ on the left-hand sides of both equations of the last remark. The condition $a \in Y$ is implied by the legitimacy of $Y \triangleleft_{a} X$ on the right-hand side of the first equation, and the condition $a \in Z$ by the legitimacy of $Z \triangleleft_{a} X$ on the right-hand side of the second equation. In the first equation we must also have that $a$ is of the form $b c$, since $b \in P_{Y}$. In the second equation this is excluded, since $Z$ is a nominal arity. 
Let $\bar{n}=d f\{1, \ldots, n\}$ for $n \geq 1$, and let $\overline{0}=\emptyset$. It is clear that $\bar{n}$ is a nominal arity for every $n \geq 0$, and $P_{\bar{n}}=\{e\}$ for $n>0$.

We pass now to the definition of the structure $\mathcal{O}_{e}$. Let $G$ and $\alpha_{G}$ be as for $\mathcal{O}$ (see the beginning of the preceding section). We define first inductively with three clauses the set of terms of $\mathcal{O}_{e}$ together with a function $s$ from these terms to nominal arities:

$\left(0_{e}\right) \quad$ if $x \in G$, then $x$ is a term; $s(x)= \begin{cases}\overline{\alpha_{G}(x)}, & \text { if } \alpha_{G}(x) \neq 1, \\ \{e\}, & \text { if } \alpha_{G}(x)=1 ;\end{cases}$

$\left(1_{e}\right) \quad \mathbf{I}$ is a term; $s(\mathbf{I})=\{e\} ;$

$\left(2_{e}\right) \quad$ if $f$ and $g$ are terms and $a \in s(g)$, then $g \triangleleft_{a} f$ is a term;

$s\left(g \triangleleft_{a} f\right)=s(g) \triangleleft_{a} a \cdot s(f)$.

(Why we do not have $s(x)=\overline{\alpha_{G}(x)}=\{1\}$ if $\alpha_{G}(x)=1$ is explained after the definition of the terms of $\mathcal{O}_{u}$ in Section 5.) We use $f, g, h, \ldots$, perhaps with indices, for the terms of $\mathcal{O}_{e}$.

Here, as for $\mathcal{O}$, the term $\mathbf{I}$ stands for the unit operadic operation, while $\triangleleft_{a}$ stands for a partial operation of insertion. The expression $g \triangleleft_{a} f$ is legitimate when $a \in s(g)$, and $f=g$ is legitimate when $s(f)=s(g)$.

The equations of $\mathcal{O}_{e}$ between terms are given as for $\mathcal{O}$ through an axiomatic equational system, whose axiomatic equations besides $f=f$ are the following equations:

(unit $_{e}$ ) $\quad f \triangleleft_{a} \mathbf{I}=f, \quad \mathbf{I} \triangleleft_{e} f=f$,

$\left(\right.$ assoc $\left.1_{e}\right) \quad\left(h \triangleleft_{b} g\right) \triangleleft_{b a} f=h \triangleleft_{b}\left(g \triangleleft_{a} f\right)$,

$\left(\right.$ assoc $\left.2_{e}\right) \quad\left(h \triangleleft_{b} g\right) \triangleleft_{a} f=\left(h \triangleleft_{a} f\right) \triangleleft_{b} g$.

As rules we have symmetry and transitivity of $=$, as for $\mathcal{O}$, and $\triangleleft_{a}$-congruence:

from $f_{1}=f_{2}$ and $g_{1}=g_{2}$ derive $g_{1} \triangleleft_{a} f_{1}=g_{2} \triangleleft_{a} f_{2}$.

This concludes our definition of the equations of $\mathcal{O}_{e}$. As for $\mathcal{O}$, the operadic operations of $\mathcal{O}_{e}$ are equivalence classes of terms such that the equations of $\mathcal{O}_{e}$ are satisfied. The structure $\mathcal{O}_{e}$ should be conceived as a partial algebra $\left\langle C\left(\mathcal{O}_{e}\right),\left\{\triangleleft_{a} \mid a\right.\right.$ is an $\mathbf{N}^{+}$-word $\left.\}, \mathbf{I}\right\rangle$, with the carrier $C\left(\mathcal{O}_{e}\right)$ of $\mathcal{O}_{e}$ being the set of operadic operations of $\mathcal{O}_{e}$.

The legitimacy of $h \triangleleft_{b} g$ on the left-hand sides of (assoc $\left.1_{e}\right)$ and $\left(\right.$ assoc $2_{e}$ ) implies $b \in s(h)$; the legitimacy of $g \triangleleft_{a} f$ on the right-hand side of (assoc $\left.1_{e}\right)$ implies $a \in s(g)$, and the legitimacy of $h \triangleleft_{a} f$ on the right-hand side of (assoc $2_{e}$ ) implies $a \in s(h)$.

When the indices of $\left(\right.$ assoc $\left.1_{e}\right)$ are compared with those of the first equation of Remark 3.4, one should bear in mind that we have the following:

$$
\begin{aligned}
s\left(\left(h \triangleleft_{b} g\right) \triangleleft_{b a} f\right) & =\left(s(h) \triangleleft_{b} b \cdot s(g)\right) \triangleleft_{b a} b a \cdot s(f), & & \text { by definition, } \\
& =s(h) \triangleleft_{b}\left(b \cdot s(g) \triangleleft_{b a} b a \cdot s(f)\right), & & \text { by Remark 3.4. } \\
& =s\left(h \triangleleft_{b}\left(g \triangleleft_{a} f\right)\right), & & \text { by definition. }
\end{aligned}
$$

Hence we have verified that for $f_{1}=f_{2}$ being an instance of (assoc $\left.1_{e}\right)$ we have $s\left(f_{1}\right)=s\left(f_{2}\right)$. This is verified analogously for the other axiomatic equations of $\mathcal{O}_{e}$, and this makes the basis of the induction on the length of derivation that shows that for every equation $f=g$ of $\mathcal{O}_{e}$ we have $s(f)=s(g)$.

We define the non-unitary structure $\mathcal{O}_{e}^{-}$like $\mathcal{O}_{e}$ save that we omit clause $\left(1_{e}\right)$ from the definition of terms, and we omit $\left(\right.$ unit $\left._{e}\right)$ from the axiomatic equations. 


\section{4. $\mathcal{O}$ AND $\mathcal{O}_{e}$}

In this section we show that $\mathcal{O}$ and $\mathcal{O}_{e}$ can be mapped one to the other in such a manner that $\mathcal{O}_{e}$ may be considered just an alternative notation for $\mathcal{O}$.

For $X$ a finite nominal arity, let the bijection $K_{X}: X \rightarrow \overline{|X|}$ be defined by

$$
K_{X}(a)=|\{b \in X \mid b \prec a\}|+1 .
$$

Note that $|\bar{n}|=n$, so that $K_{\bar{n}}$ is a function from $\bar{n}$ to $\bar{n}$.

It is easy to see that $a_{1} \prec a_{2}$ implies $K_{X}\left(a_{1}\right)<K_{X}\left(a_{2}\right)$, provided $a_{1}, a_{2} \in X$. It is also easy to verify the following:

(K1) $\quad K_{\bar{n}}$ is the identity function from $\bar{n}$ to $\bar{n}, \quad K_{\{e\}}(e)=1$,

$(K 2) \quad K_{b \cdot X}(b a)=K_{X}(a)$,

$(K 3.1) \quad K_{Y \triangleleft_{b} X}(a)=K_{Y}(b)-1+K_{X}(a), \quad$ if $a \in X$,

$(K 3.2) \quad K_{Y \triangleleft_{b} X}(a)=K_{Y}(a)$, if $a \in Y$ and $a \prec b$,

(K3.3) $K_{Y \triangleleft_{b} X}(a)=K_{Y}(a)-1+|X|, \quad$ if $a \in Y$ and $b \prec a$.

Let $K_{X}^{-1}: \overline{|X|} \rightarrow X$ be the bijection inverse to $K_{X}$. It is easy to verify for $K_{X}^{-1}$ the following equations, which are interdeducible with those we have just given for $K_{X}$ :

$\left(K^{-1} 1\right) \quad K_{\bar{n}}^{-1}=K_{\bar{n}}, \quad K_{\{e\}}^{-1}(1)=e$,

$\left(K^{-1} 2\right) \quad K_{b \cdot X}^{-1}(n)=b K_{X}^{-1}(n), \quad$ for $n \in \overline{|X|}$,

$\left(K^{-1} 3.1\right) \quad K_{Y \triangleleft_{b} X}^{-1}(m)=K_{X}^{-1}(m-n+1)$, if $K_{Y}(b)=n$ and $n \leq m<n+|X|$,

$\left(K^{-1} 3.2\right) \quad K_{Y \triangleleft_{b} X}^{-1}(m)=K_{Y}^{-1}(m), \quad$ if $m<K_{Y}(b)$,

$\left(K^{-1} 3.3\right) \quad K_{Y \triangleleft_{b} X}^{-1}(m)=K_{Y}^{-1}(m-|X|+1), \quad$ if $K_{Y}(b)+|X| \leq m$.

These equations and those given above for $K_{X}$ serve to explain how we pass from the indices in the axiomatic equations of $\mathcal{O}$ to those in the axiomatic equations of $\mathcal{O}_{e}$, and vice versa. In particular the condition of $\left(K^{-1} 3.1\right)$ is transferred to (assoc 1$)$, while the conditions of $\left(K^{-1} 3.2\right)$ and $\left(K^{-1} 3.3\right)$ are transferred to (assoc 2$)$.

Next we define inductively a map $\varepsilon$ from the terms of $\mathcal{O}$ to the terms of $\mathcal{O}_{e}$, for which we will show below (in Propositions 4.1 and 4.2) that it is a bijection:

$$
\begin{aligned}
& \varepsilon(x)=x, \quad \varepsilon(\mathbf{I})=\mathbf{I}, \\
& \varepsilon\left(\gamma \triangleleft_{n} \varphi\right)=\varepsilon(\gamma) \triangleleft_{a} \varepsilon(\phi), \text { for } K_{s(\varepsilon(\gamma))}^{-1}(n)=a .
\end{aligned}
$$

We define inductively the map $\tau$ from the terms of $\mathcal{O}_{e}$ to the terms of $\mathcal{O}$, for which we will show that it is the inverse of $\varepsilon$ :

$$
\begin{aligned}
& \tau(x)=x, \quad \tau(\mathbf{I})=\mathbf{I}, \\
& \tau\left(g \triangleleft_{a} f\right)=\tau(g) \triangleleft_{n} \tau(f), \quad \text { for } K_{s(g)}(a)=n .
\end{aligned}
$$

Then we can establish the following propositions by straightforward inductions on the complexity of $\varphi$ and $f$.

Proposition 4.1. For every term $\varphi$ of $\mathcal{O}$ we have that $\tau(\varepsilon(\varphi))$ is $\varphi$.

Proposition 4.2. For every term $f$ of $\mathcal{O}_{e}$ we have that $\varepsilon(\tau(f))$ is $f$.

By inductions on the complexity of $\varphi$ and $f$ we can also straightforwardly establish the following lemmata. 
Lemma 4.3. For every term $\varphi$ of $\mathcal{O}$ we have $|s(\varepsilon(\varphi))|=\alpha(\varphi)$.

Lemma 4.4. For every term $f$ of $\mathcal{O}_{e}$ we have $\alpha(\tau(f))=|s(f)|$.

These lemmata are used in the proof of the following two propositions, which consist in inductions on the length of derivation. The main part of these inductions is however in the basis, when we deal with axiomatic equations.

Proposition 4.5. If $\varphi=\gamma$ in $\mathcal{O}$, then $\varepsilon(\varphi)=\varepsilon(\gamma)$ in $\mathcal{O}_{e}$.

Proposition 4.6. If $f=g$ in $\mathcal{O}_{e}$, then $\tau(f)=\tau(g)$ in $\mathcal{O}$.

So $\varepsilon$ and $\tau$ induce bijections inverse to each other between the operadic operations of $\mathcal{O}$ and $\mathcal{O}_{e}$. This shows that $\mathcal{O}_{e}$ is just a notational variant of $\mathcal{O}$.

The bijections between the terms of $\mathcal{O}^{-}$and $\mathcal{O}_{e}^{-}$are obtained by just restricting $\varepsilon$ and $\tau$, and then for these bijections we can establish as well as Propositions 4.1 and 4.2 the analogues of Propositions 4.5 and 4.6, where $\mathcal{O}$ and $\mathcal{O}_{e}$ are replaced respectively by $\mathcal{O}^{-}$ and $\mathcal{O}_{e}^{-}$. So the relationship between $\mathcal{O}^{-}$and $\mathcal{O}_{e}^{-}$is exactly analogous to that between $\mathcal{O}$ and $\mathcal{O}_{e}$.

\section{The Structure $\mathcal{O}_{u}$}

We introduce now the structure $\mathcal{O}_{u}$, which generalizes $\mathcal{O}_{e}$.

Let $G$ and $\alpha_{G}$ be as for $\mathcal{O}$ and $\mathcal{O}_{e}$ (see the beginning of Section 2). We define first inductively with three clauses the set of terms of $\mathcal{O}_{u}$ together with a function $s$ from these terms to nominal arities (this function is related to the function $s$ from the terms of $\mathcal{O}_{e}$, and this is why it bears the same name) and a function $t$ from these terms to $\mathbf{N}^{+}$-words:

$\left(0_{u}\right) \quad$ if $x \in G$ and $a$ is an $\mathbf{N}^{+}$-word, then $a \cdot x$ is a term;

$$
s(a \cdot x)=\left\{\begin{array}{ll}
a \cdot \overline{\alpha_{G}(x)}, & \text { if } \alpha_{G}(x) \neq 1, \\
\{a\}, & \text { if } \alpha_{G}(x)=1,
\end{array} \quad t(a \cdot x)=a ;\right.
$$

$\left(1_{u}\right) \quad$ if $a$ is an $\mathbf{N}^{+}$-word, then $a \cdot \mathbf{I}$ is a term; $s(a \cdot \mathbf{I})=\{a\}, \quad t(a \cdot \mathbf{I})=a$;

$\left(2_{u}\right) \quad$ if $f$ and $g$ are terms and $t(f) \in s(g)$, then $g \triangleleft f$ is a term; $s(g \triangleleft f)=s(g) \triangleleft_{t(f)} s(f), \quad t(g \triangleleft f)=t(g)$.

If $\alpha_{G}(x)=1$, then we can envisage having $s(a \cdot x)=a \cdot \overline{\alpha_{G}(x)}=\{a 1\}$ (which would entail $s(x)=\overline{\alpha_{G}(x)}=\{1\}$ for $\mathcal{O}_{e}$ ). This way diversification (see Section 1) would apply also to the unary members of $\Gamma$, but a difference would arise with the treatment of $\mathbf{I}$, which must have the clause above. We have preferred however not to distinguish these two unary cases, because this is not essential. The diversification we achieve is sufficient for our purposes (in particular for Section 18).

In order to verify for $\left(2_{u}\right)$ that $s(g) \triangleleft_{t(f)} s(f)$ is legitimate if $t(f) \in s(g)$, we have first that $t(a \cdot x) \in P_{s(a \cdot x)}$ and $t(a \cdot \mathbf{I}) \in P_{s(a \cdot \mathbf{I})}$, and we have the following two remarks.

Remark 5.1. If $t(f) \in P_{s(f)}$ and $t(f) \in s(g)$, then $s(g) \triangleleft_{t(f)} s(f)$ is legitimate.

Remark 5.2. If $t(g) \in P_{s(g)}$, then $t(g \triangleleft f) \in P_{s(g \triangleleft f)}$.

To justify the last remark we have

$$
t(g \triangleleft f)=t(g) \in P_{s(g)} \subseteq P_{s(g) \triangleleft} \triangleleft_{t(f)} s(f)
$$


by Remark 3.2. These two remarks, together with what we said before them for the basis of the induction, yield that $t(f) \in P_{s(f)}$ holds for every term $f$ of $\mathcal{O}_{u}$.

This concludes our definition of the terms of $\mathcal{O}_{u}$. We use now $f, g, h, \ldots$, perhaps with indices, for the terms of $\mathcal{O}_{u}$. The expression $g \triangleleft f$ is legitimate when $t(f) \in s(g)$, and $f=g$ is legitimate when $s(f)=s(g)$ and $t(f)=t(g)$.

The equations of $\mathcal{O}_{u}$ between terms are given as before through an axiomatic equational system, whose axiomatic equations besides $f=f$ are the following equations:

( unit $\left._{u}\right) \quad f \triangleleft a \cdot \mathbf{I}=f, \quad t(f) \cdot \mathbf{I} \triangleleft f=f$,

$\left(\right.$ assoc $\left.1_{u}\right) \quad(h \triangleleft g) \triangleleft f=h \triangleleft(g \triangleleft f)$,

$($ assoc $2 u) \quad(h \triangleleft g) \triangleleft f=(h \triangleleft f) \triangleleft g$.

As rules we have symmetry and transitivity of $=$, and $\triangleleft$-congruence, which is like $\triangleleft_{a^{-}}$ congruence of $\mathcal{O}_{e}$ with the subscript $a$ omitted.

This concludes our definition of the equations of $\mathcal{O}_{u}$, and of the operadic operations of $\mathcal{O}_{u}$ (which, as before, are equivalence classes of terms). The structure $\mathcal{O}_{u}$ should be conceived as a partial algebra $\left\langle C\left(\mathcal{O}_{u}\right), \triangleleft,\left\{a \cdot \mathbf{I} \mid a\right.\right.$ is an $\mathbf{N}^{+}$-word $\left.\}\right\rangle$, with the carrier $C\left(\mathcal{O}_{u}\right)$ of $\mathcal{O}_{u}$ being the set of operadic operations of $\mathcal{O}_{u}$.

The legitimacy of $h \triangleleft g$ on the left-hand sides of $\left(\right.$ assoc $1_{u}$ ) and (assoc $2_{u}$ ) implies $t(g) \in s(h)$, while the legitimacy of $g \triangleleft f$ on the right-hand side of (assoc $1_{u}$ ) implies $t(f) \in s(g)$, and the legitimacy of $h \triangleleft f$ on the right-hand side of (assoc $2_{u}$ ) implies $t(f) \in s(h)$. By induction on the length of derivation we establish that for every equation $f=g$ of $\mathcal{O}_{u}$ we have $s(f)=s(g)$ and $t(f)=t(g)$.

We define the non-unitary structure $\mathcal{O}_{u}^{-}$like $\mathcal{O}_{u}$ save that we omit clause $\left(1_{u}\right)$ from the definition of terms, and we omit $\left(u_{i n} t_{u}\right)$ from the axiomatic equations.

The structure $\mathcal{O}_{u}$ amounts to the free multicategory generated by the multigraph made of the objects in $\left\{a \mid a\right.$ is an $\mathbf{N}^{+}$-word $\}$and the multiarrows in $\{a \cdot x \mid x \in G$ and $a$ is an $\mathbf{N}^{+}$-word , with the source and target functions given by the functions $s$ and $t$ (see [Lam89, Section 3).

\section{6. $\mathcal{O}_{e}$ AND $\mathcal{O}_{u}$}

In this section we establish the correspondences that exist between $\mathcal{O}_{e}$ and some structures derived form $\mathcal{O}_{u}$.

For every $\mathbf{N}^{+}$-word $a$ we define inductively a map $a$. from the terms of $\mathcal{O}_{u}$ to the terms of $\mathcal{O}_{u}$ :

$$
\begin{aligned}
& a \cdot(b \cdot x)=a b \cdot x, \quad a \cdot(b \cdot \mathbf{I})=a b \cdot \mathbf{I}, \\
& a \cdot(g \triangleleft f)=a \cdot g \triangleleft a \cdot f ;
\end{aligned}
$$

$a \cdot f$ stands for $a \cdot(f)$, and we read $a \cdot g \triangleleft a \cdot f$ as $(a \cdot g) \triangleleft(a \cdot f)$. In order to verify that $a \cdot g \triangleleft a \cdot f$ is legitimate, we need to show that $t(a \cdot f) \in s(a \cdot g)$. For that we establish first that we have

$$
(\text { st } a \cdot) \quad s(a \cdot h)=a \cdot s(h), \quad t(a \cdot h)=a t(h)
$$

for $h$ being $b \cdot x$ and $b \cdot \mathbf{I}$ (we use here the first equation of Remark 3.3). Next we have the following two remarks.

Remark 6.1. If $t(a \cdot f)=a t(f), s(a \cdot g)=a \cdot s(g)$ and $t(f) \in s(g)$, then $t(a \cdot f) \in s(a \cdot g)$.

Remark 6.2. If (st $a$ ) holds for $h$ being $f$ and $g$, then it holds for $h$ being $g \triangleleft f$. 
The last remark (for which we use the second equation of Remark 3.3) is the induction step, which together with what we said above for the basis of the induction, yields that (st $a \cdot$ ) holds for every term $h$ of $\mathcal{O}_{u}$.

It is easy to infer that for every term $f$ of $\mathcal{O}_{u}$ we have $e \cdot f=f$ and $a \cdot(b \cdot f)=a b \cdot f$. We can also easily establish the following by induction on the length of derivation.

Remark 6.3. If $f=g$ in $\mathcal{O}_{u}$, then $a \cdot f=a \cdot g$ in $\mathcal{O}_{u}$.

So $a$. induces a map from the operadic operations of $\mathcal{O}_{u}$ to the operadic operations of $\mathcal{O}_{u}$.

We need $a$. to define inductively a map $U$ from the terms of $\mathcal{O}_{e}$ to the terms of $\mathcal{O}_{u}$ :

$$
\begin{aligned}
& U(x)=e \cdot x, \quad U(\mathbf{I})=e \cdot \mathbf{I}, \\
& U\left(g \triangleleft_{a} f\right)=U(g) \triangleleft a \cdot U(f) .
\end{aligned}
$$

In order to verify that $U(g) \triangleleft a \cdot U(f)$ is legitimate if $g \triangleleft_{a} f$ is legitimate, we need to show that $t(a \cdot U(f)) \in s(U(g))$ follows from $a \in s(g)$. For that we rely on $($ st $a \cdot)$ and on

$$
(s t U) \quad s(U(h))=s(h), \quad t(U(h))=e,
$$

where $h$ is $g$ or $f$, as needed. It is clear that (st $U$ ) holds for $h$ being $x$ and $\mathbf{I}$, and then we may establish the induction step, which yields that $(s t U)$ holds for every term $h$ of $\mathcal{O}_{e}$.

If $c$ is the $\mathbf{N}^{+}$-word $a b$, then $a \backslash c$ is defined, and is $b$; i.e. $a \backslash a b=b$. Since $c$ is $e c$, we have that $e \backslash c$ is always defined, and is $c$.

Then we define inductively a map $E$ from the terms of $\mathcal{O}_{u}$ to the terms of $\mathcal{O}_{e}$ :

$$
\begin{aligned}
& E(a \cdot x)=x, \quad E(a \cdot \mathbf{I})=\mathbf{I}, \\
& E(g \triangleleft f)=E(g) \triangleleft_{t(g) \backslash t(f)} E(f) .
\end{aligned}
$$

We have that $t(f)$ in the last line is of the form $t(g) b$ because $t(g) \in P_{s(g)}$ and $t(f) \in$ $s(g)$. In order to verify that $t(g) \backslash t(f) \in s(E(g))$, which we need for the legitimacy of $E(g) \triangleleft_{t(g) \backslash t(f)} E(f)$, we rely on

$$
(\text { st } E) \quad t(h) \cdot s(E(h))=s(h)
$$

where $h$ is $g$. It is clear that (st $E$ ) holds for $h$ being $a \cdot x$ and $a \cdot \mathbf{I}$, and then we may establish the induction step, which yields that (st $E$ ) holds for every term $h$ of $\mathcal{O}_{u}$.

With the help of $(s t a \cdot)$, it is easy to establish the following by induction on the complexity of $f$.

Lemma 6.4. For every term $f$ of $\mathcal{O}_{u}$ we have that $E(a \cdot f)$ is $E(f)$.

This lemma, together with $(s t a \cdot)$ and $(s t U)$, serves for the first of the following two propositions, which are proved by inductions on the complexity of $f$.

Proposition 6.5. For every term $f$ of $\mathcal{O}_{e}$ we have that $E(U(f))$ is $f$.

Proposition 6.6. For every term $f$ of $\mathcal{O}_{u}$ we have that $t(f) \cdot U(E(f))$ is $f$.

Next we establish the following two propositions by inductions on the length of derivation.

Proposition 6.7. If $f=g$ in $\mathcal{O}_{e}$, then $U(f)=U(g)$ in $\mathcal{O}_{u}$.

Proposition 6.8. If $f=g$ in $\mathcal{O}_{u}$, then $E(f)=E(g)$ in $\mathcal{O}_{e}$.

The only case that is perhaps not quite straightforward is with $\left(\right.$ assoc $\left.1_{u}\right)$ in the basis of the induction in the proof of Proposition 6.8. Here is how we proceed in that case:

$$
\begin{aligned}
& E((h \triangleleft g) \triangleleft f)=\left(E(h) \triangleleft_{t(h) \backslash t(g)} E(g)\right) \triangleleft_{t(h) \backslash t(f)} E(f), \\
& E(h \triangleleft(g \triangleleft f))=E(h) \triangleleft_{t(h) \backslash t(g)}\left(E(g) \triangleleft_{t(g) \backslash t(f)} E(f)\right) .
\end{aligned}
$$


Since we have that $t(f)$ is of the form $t(h) b a$, where $t(g)$ is $t(h) b$, we may apply $\left(\right.$ assoc $\left.1_{e}\right)$.

As corollaries of the four propositions just established, we have the following.

Proposition 6.9. $f=g$ in $\mathcal{O}_{e}$ iff $U(f)=U(g)$ in $\mathcal{O}_{u}$.

Proposition 6.10. If $t(f)=t(g)$, then $f=g$ in $\mathcal{O}_{u}$ iff $E(f)=E(g)$ in $\mathcal{O}_{e}$.

Consider the following set of operadic operations of $\mathcal{O}_{u}$ for a given $\mathbf{N}^{+}$-word $b$ :

$$
C\left(\mathcal{O}_{u}(b)\right)={ }_{d f}\left\{f \in C\left(\mathcal{O}_{u}\right) \mid t(f)=b\right\},
$$

and consider the structure

$$
\mathcal{O}_{u}(b)={ }_{d f}\left\langle C\left(\mathcal{O}_{u}(b)\right),\left\{\triangleleft a \cdot \mid a \text { is an } \mathbf{N}^{+} \text {-word }\right\}, b \cdot \mathbf{I}\right\rangle .
$$

The operation $\triangleleft a$. is the partial operation on operadic operations defined in $\mathcal{O}_{u}$, which applied to $f$ and $g$ yields $g \triangleleft a \cdot f$, provided $a t(f) \in s(g)$. We can prove the following.

Proposition 6.11. The structures $\mathcal{O}_{e}$ and $\mathcal{O}_{u}(e)$ are isomorphic.

Proof. Propositions 6.56.8 show that, on the one hand, the map $U$ and, on the other hand, the map $E$ restricted to the terms of $\mathcal{O}_{u}(e)$ (i.e. those terms of $\mathcal{O}_{u}$ that stand for the members of $\left.C\left(\mathcal{O}_{u}(e)\right)\right)$ induce bijections inverse to each other between $C\left(\mathcal{O}_{e}\right)$ and $C\left(\mathcal{O}_{u}(e)\right)$. It remains only to consider the definition of $U$ to establish that $U$, and hence $E$ too, are homomorphisms.

For a given $\mathbf{N}^{+}$-word $b$ let

$$
\begin{aligned}
C\left(\mathcal{O}_{u}(b \cdot)\right) & ={ }_{d f}\left\{f \in C\left(\mathcal{O}_{u}\right) \mid t(f)=b a \text { for some } \mathbf{N}^{+} \text {-word } a\right\} \\
& =\cup\left\{C\left(\mathcal{O}_{u}(b a)\right) \mid a \text { is an } \mathbf{N}^{+} \text {-word }\right\},
\end{aligned}
$$

and consider the structure

$$
\mathcal{O}_{u}(b \cdot)=_{d f}\left\langle C\left(\mathcal{O}_{u}(b \cdot)\right), \triangleleft,\left\{b a \cdot \mathbf{I} \mid a \text { is an } \mathbf{N}^{+} \text {-word }\right\}\right\rangle .
$$

Note that $\mathcal{O}_{u}(e \cdot)$ is $\mathcal{O}_{u}$.

We define inductively a map $b \backslash$ from $C\left(\mathcal{O}_{u}(b \cdot)\right)$ to $C\left(\mathcal{O}_{u}\right)$ by

$$
\begin{aligned}
& b \backslash(b a \cdot x)=a \cdot x, \quad b \backslash(b a \cdot \mathbf{I})=a \cdot \mathbf{I}, \\
& b \backslash(g \triangleleft f)=b \backslash g \triangleleft b \backslash f ;
\end{aligned}
$$

$b \backslash f$ stands for $b \backslash(f)$, and we read $b \backslash g \triangleleft b \backslash f$ as $(b \backslash g) \triangleleft(b \backslash f)$. In the last clause, we have that $b \backslash f$ is defined because $t(f) \in s(g)$ and $t(g) \in P_{s(g)}$. We can prove the following.

Proposition 6.12. For every $\mathbf{N}^{+}$-word $b$ the structures $\mathcal{O}_{u}$ and $\mathcal{O}_{u}(b \cdot)$ are isomorphic.

Proof. From $\mathcal{O}_{u}$ to $\mathcal{O}_{u}(b \cdot)$ we have the map $b \cdot$, and it is easy to show that $b \backslash$ is its inverse.

Let $\mathcal{O}_{u}^{\triangleleft}(b)$ be the structure $\left\langle C\left(\mathcal{O}_{u}(b)\right), \triangleleft, b \cdot \mathbf{I}\right\rangle$, which is a substructure of $\mathcal{O}_{u}(b)$ (from the family of insertions $\left\{\triangleleft a \cdot \mid a\right.$ is an $\mathbf{N}^{+}$-word $\}$we keep only $\triangleleft e \cdot$, which amounts to $\left.\triangleleft\right)$. It is also a substructure of $\mathcal{O}_{u}(b \cdot)$, and is a kind of common denominator of $\mathcal{O}_{u}(b)$ and $\mathcal{O}_{u}(b \cdot)$. We can prove the following.

Proposition 6.13. For all $\mathbf{N}^{+}$-words $a$ and $b$ the structures $\mathcal{O}_{u}^{\triangleleft}(a)$ and $\mathcal{O}_{u}^{\triangleleft}(b a)$ are isomorphic.

Proof. We restrict the maps $b$ and $b \backslash$, which we used for the proof of the preceding proposition. 
As a corollary of this proposition we obtain that for every $\mathbf{N}^{+}$-word $b$ the structures $\mathcal{O}_{u}^{\triangleleft}(e)$ and $\mathcal{O}_{u}^{\triangleleft}(b)$ are isomorphic.

Everything we said in this section about the relationship between $\mathcal{O}_{e}$ and $\mathcal{O}_{u}$ can be restricted to the non-unitary structures $\mathcal{O}_{e}^{-}$and $\mathcal{O}_{u}^{-}$.

\section{PART II}

\section{The Category $\mathcal{W O}_{u}^{-}$}

We introduce a category that will be a weakened version of $\mathcal{O}_{u}$, with axiomatic equations replaced by isomorphisms. We deal first in this section with the non-unitary category, and add what is required for the unitary category in the next section. We deal in these two sections with the $u$ versions of the weakened notions, leaving for sections 9-10 the more complicated $e$ versions.

The object of $\mathcal{W} \mathcal{O}_{u}^{-}$are the terms of $\mathcal{O}_{u}^{-}$(not the operadic operations of $\mathcal{O}_{u}^{-}$; see Section 5). We define inductively the arrow terms of $\mathcal{W O}_{u}^{-}$. Every arrow term has a type, which is a pair of objects $(f, g)$; as usual, we write $u: f \rightarrow g$ to indicate that the arrow term $u$ is of that type. The object $f$ is the source, and $g$ the target, of $u$. We specify first the basic arrow terms:

$$
\begin{aligned}
& \mathbf{1}_{f}: f \rightarrow f, \\
& \beta_{h, g, f}:(h \triangleleft g) \triangleleft f \rightarrow h \triangleleft(g \triangleleft f), \quad \beta_{h, g, f}^{-1}: h \triangleleft(g \triangleleft f) \rightarrow(h \triangleleft g) \triangleleft f, \\
& \theta_{h, g, f}:(h \triangleleft g) \triangleleft f \rightarrow(h \triangleleft f) \triangleleft g .
\end{aligned}
$$

We can make for these arrow terms comments on the legitimacy of expressions for objects in their types exactly analogous to those made for the equations (assoc $1_{u}$ ) and (assoc $2_{u}$ ) in Section 5, from which these arrow terms are derived.

Next we have the following two partial operations on arrow terms:

if $u: f \rightarrow f^{\prime}$ and $v: g \rightarrow g^{\prime}$ are arrow terms, then $v \circ u: f \rightarrow g^{\prime}$ is an arrow term when $f^{\prime}$ is $g$, and $v \triangleleft u: g \triangleleft f \rightarrow g^{\prime} \triangleleft f^{\prime}$ is an arrow term when $g \triangleleft f$ and $g^{\prime} \triangleleft f^{\prime}$ are legitimate.

This concludes the definition of the arrow terms of $\mathcal{W O}_{u}^{-}$. We use $u, v, w, \ldots$, perhaps with indices, for arrow terms.

Note that $\triangleleft$ occurs now on three levels: first, at the level of nominal arities in $Y \triangleleft_{a} X$, which underly the objects, secondly, at the level of objects in $g \triangleleft f$, which underly the arrow terms, and thirdly, at the level of arrow terms in $v \triangleleft u$.

Since the arrow terms of $\mathcal{W O}_{u}^{-}$are derived from the equations of $\mathcal{O}_{u}^{-}$, we obtain immediately from what we established by induction on the length of derivation for $\mathcal{O}_{u}^{-}$in Section 5 that for every arrow term $u: f \rightarrow g$ of $\mathcal{W} \mathcal{O}_{u}^{-}$we have $s(f)=s(g)$ and $t(f)=t(g)$.

The equations of $\mathcal{W} \mathcal{O}_{u}^{-}$between arrow terms are given through an axiomatic equational system, and the arrows of $\mathcal{W} \mathcal{O}_{u}^{-}$will be formally equivalence classes of arrow terms such that these equations are satisfied. Besides $u=u$ and the categorial equations $u \circ \mathbf{1}_{f}=u=\mathbf{1}_{g} \circ u$, for $u: f \rightarrow g$, and $(w \circ v) \circ u=w \circ(v \circ u)$, the axiomatic equations of $\mathcal{W} \mathcal{O}_{u}^{-}$are the following: (ins 1) $\mathbf{1}_{g} \triangleleft \mathbf{1}_{f}=\mathbf{1}_{g \triangleleft f}$,

(ins 2) $\left(v_{2} \circ v_{1}\right) \triangleleft\left(u_{2} \circ u_{1}\right)=\left(v_{2} \triangleleft u_{2}\right) \circ\left(v_{1} \triangleleft u_{1}\right)$,

$(\beta$ nat $) \quad \beta_{h_{2}, g_{2}, f_{2}} \circ((w \triangleleft v) \triangleleft u)=(w \triangleleft(v \triangleleft u)) \circ \beta_{h_{1}, g_{1}, f_{1}}$,

$(\theta$ nat $) \quad \theta_{h_{2}, g_{2}, f_{2}} \circ((w \triangleleft v) \triangleleft u)=((w \triangleleft u) \triangleleft v) \circ \theta_{h_{1}, g_{1}, f_{1}}$, 


$$
\begin{array}{ll}
(\beta \beta) & \beta_{h, g, f}^{-1} \circ \beta_{h, g, f}=\mathbf{1}_{(h \triangleleft g) \triangleleft f}, \quad \beta_{h, g, f} \circ \beta_{h, g, f}^{-1}=\mathbf{1}_{h \triangleleft(g \triangleleft f)}, \\
(\theta \theta) & \theta_{h, f, g} \circ \theta_{h, g, f}=\mathbf{1}_{(h \triangleleft g) \triangleleft f}, \\
(\beta \text { pent }) & \left(\mathbf{1}_{j} \triangleleft \beta_{h, g, f}\right) \circ \beta_{j, h \triangleleft g, f} \circ\left(\beta_{j, h, g} \triangleleft \mathbf{1}_{f}\right)=\beta_{j, h, g \triangleleft f} \circ \beta_{j \triangleleft h, g, f}, \\
(\theta \text { YB }) & \theta_{j \triangleleft f, h, g} \circ\left(\theta_{j, h, f} \triangleleft \mathbf{1}_{g}\right) \circ \theta_{j \triangleleft h, g, f}=\left(\theta_{j, g, f} \triangleleft \mathbf{1}_{h}\right) \circ \theta_{j \triangleleft g, h, f} \circ\left(\theta_{j, h, g} \triangleleft \mathbf{1}_{f}\right), \\
(\beta \theta 1) & \left(\mathbf{1}_{j} \triangleleft \theta_{h, g, f} \circ \beta_{j, h \triangleleft g, f} \circ\left(\beta_{j, h, g} \triangleleft \mathbf{1}_{f}\right)=\beta_{j, h \triangleleft f, g} \circ\left(\beta_{j, h, f} \triangleleft \mathbf{1}_{g}\right) \circ \theta_{j \triangleleft h, g, f},\right. \\
(\beta \theta 2) & \theta_{j, h \triangleleft g, f} \circ\left(\beta_{j, h, g} \triangleleft \mathbf{1}_{f}\right)=\beta_{j \triangleleft f, h, g} \circ\left(\theta_{j, h, f} \triangleleft \mathbf{1}_{g}\right) \circ \theta_{j \triangleleft h, g, f} .
\end{array}
$$

The name of ( $\beta$ pent) comes from Mac Lane's pentagon of monoidal categories (see MLa63 and MLa98, Section VII.1), while ( $\theta$ YB) is related to the equation (YB) of Section 16 (and YB comes from Yang-Baxter).

As rules we have symmetry and transitivity of $=$, and for $\xi$ being $\circ$ and $\triangleleft$ the congruence rules:

$$
\text { from } u_{1}=u_{2} \text { and } v_{1}=v_{2} \text { derive } v_{1} \xi u_{1}=v_{2} \xi u_{2} \text {. }
$$

This concludes our definition of the equations of $\mathcal{W} \mathcal{O}_{u}^{-}$, and of the category $\mathcal{W} \mathcal{O}_{u}^{-}$.

An equation between the arrow terms of a category is legitimate when both sides are of the same type, and one can easily check by induction on the length of derivation that the equations of our axiomatic system for $\mathcal{W O}_{u}^{-}$satisfy this requirement for legitimacy. The same holds for the equational axiomatic system of all the categories introduced later, and we will not mention this matter any more.

\section{The CATEGory $\mathcal{W O}_{u}$}

We add now to $\mathcal{W O}_{u}^{-}$what is needed to obtain the unitary category $\mathcal{W O}_{u}$.

The objects of $\mathcal{W O}_{u}$ are the terms of $\mathcal{O}_{u}$ (see Section 5). The arrow terms of $\mathcal{W} \mathcal{O}_{u}$ are defined like those of $\mathcal{W O}_{u}^{-}$in the preceding section with the following additional basic arrow terms, derived from the equations $\left(u_{n i t}\right)$ of Section 5 :

$$
\begin{aligned}
& \mu_{f, a}: f \triangleleft a \cdot \mathbf{I} \rightarrow f, \quad \mu_{f, a}^{-1}: f \rightarrow f \triangleleft a \cdot \mathbf{I}, \\
& \lambda_{f}: t(f) \cdot \mathbf{I} \triangleleft f \rightarrow f, \quad \lambda_{f}^{-1}: f \rightarrow t(f) \cdot \mathbf{I} \triangleleft f .
\end{aligned}
$$

The equation of $\mathcal{W} \mathcal{O}_{u}$ between arrow terms are defined like those of $\mathcal{W} \mathcal{O}_{u}^{-}$in the preceding section with the following additional axiomatic equations:

( $\mu$ nat) $\quad \mu_{f_{2}, a} \circ\left(u \triangleleft \mathbf{1}_{a \cdot \mathbf{I}}\right)=u \circ \mu_{f_{1}, a}$,

$(\lambda$ nat $) \quad \lambda_{f_{2}} \circ\left(\mathbf{1}_{t\left(f_{1}\right) \cdot \mathbf{I}} \triangleleft u\right)=u \circ \lambda_{f_{1}}$,

$(\mu \mu) \quad \mu_{f, a}^{-1} \circ \mu_{f, a}=\mathbf{1}_{f \triangleleft a \cdot \mathbf{I}}, \quad \mu_{f, a} \circ \mu_{f, a}^{-1}=\mathbf{1}_{f}$,

$(\lambda \lambda) \quad \lambda_{f}^{-1} \circ \lambda_{f}=\mathbf{1}_{t(f) \cdot \mathbf{I} \triangleleft f}, \quad \lambda_{f} \circ \lambda_{f}^{-1}=\mathbf{1}_{f}$,

$(\beta \mu \lambda) \quad \beta_{h, t(f) \cdot \mathbf{I}, f}=\left(\mathbf{1}_{h} \triangleleft \lambda_{f}^{-1}\right) \circ\left(\mu_{h, t(f)} \triangleleft \mathbf{1}_{f}\right)$,

$(\theta \mu) \quad \theta_{h, b \cdot \mathbf{I}, f}=\mu_{h \triangleleft f, b}^{-1} \circ\left(\mu_{h, b} \triangleleft \mathbf{1}_{f}\right)$. 


\section{The Category $\mathcal{W O}_{e}^{-}$}

We introduce in this section the $e$ analogue of the non-unitary category $\mathcal{W O}_{u}^{-}$of Section 7 .

The object of $\mathcal{W O}_{e}^{-}$are the terms of $\mathcal{O}_{e}^{-}$(see Section 3). To define inductively the arrow terms of $\mathcal{W O}_{e}^{-}$, we specify first the basic arrow terms:

$$
\begin{aligned}
& \mathbf{1}_{f}: f \rightarrow f, \\
& \beta_{h,(b, g),(a, f)}:\left(h \triangleleft_{b} g\right) \triangleleft_{b a} f \rightarrow h \triangleleft_{b}\left(g \triangleleft_{a} f\right), \\
& \beta_{h,(b, g),(a, f)}^{-1}: h \triangleleft_{b}\left(g \triangleleft_{a} f\right) \rightarrow\left(h \triangleleft_{b} g\right) \triangleleft_{b a} f, \\
& \theta_{h,(b, g),(a, f)}:\left(h \triangleleft_{b} g\right) \triangleleft_{a} f \rightarrow\left(h \triangleleft_{a} f\right) \triangleleft_{b} g .
\end{aligned}
$$

We can make for these arrow terms comments on the legitimacy of expressions for objects in their types exactly analogous to those made for the equations (assoc $\left.1_{e}\right)$ and $\left(\operatorname{assoc} 22_{e}\right)$ in Section 3, from which these arrow terms are derived.

The operations under which the arrow terms are closed are composition $\circ$ and the operations $\triangleleft_{a}$ for which we have the following clause:

if $u: f \rightarrow f^{\prime}$ and $v: g \rightarrow g^{\prime}$ are arrow terms, then $v \triangleleft_{a} u: g \triangleleft_{a} f \rightarrow g^{\prime} \triangleleft_{a} f^{\prime}$ is an arrow term, when $g \triangleleft_{a} f$ and $g^{\prime} \triangleleft_{a} f^{\prime}$ are legitimate.

This concludes the definition of the arrow terms of $\mathcal{W O}_{e}^{-}$. We use still $u, v, w, \ldots$, perhaps with indices, for these newly introduced arrow terms. It follows immediately from what we established for $\mathcal{O}_{e}^{-}$in Section 3 that for every arrow term $u: f \rightarrow g$ of $\mathcal{W O}_{e}^{-}$we have $s(f)=s(g)$.

The equations of $\mathcal{W O}_{e}^{-}$between arrow terms are given through an axiomatic equational system, which besides $u=u$ and the categorial equations (as those given in Section 7 for $\mathcal{W} \mathcal{O}_{u}^{-}$) has the following axiomatic equations:

$$
\begin{aligned}
& \left(\text { ins } 1_{e}\right) \quad \mathbf{1}_{g} \triangleleft_{a} \mathbf{1}_{f}=\mathbf{1}_{g \triangleleft_{a} f} \text {, } \\
& \left(\text { ins } 2_{e}\right) \quad\left(v_{2} \circ v_{1}\right) \triangleleft_{a}\left(u_{2} \circ u_{1}\right)=\left(v_{2} \triangleleft_{a} u_{2}\right) \circ\left(v_{1} \triangleleft_{a} u_{1}\right) \text {, } \\
& \left(\beta \text { nat }_{e}\right) \quad \beta_{h_{2},\left(b, g_{2}\right),\left(a, f_{2}\right)} \circ\left(\left(w \triangleleft_{b} v\right) \triangleleft_{b a} u\right)=\left(w \triangleleft_{b}\left(v \triangleleft_{a} u\right)\right) \circ \beta_{h_{1},\left(b, g_{1}\right),\left(a, f_{1}\right)}, \\
& \left(\theta \text { nat }_{e}\right) \quad \theta_{h_{2},\left(b, g_{2}\right),\left(a, f_{2}\right)} \circ\left(\left(w \triangleleft_{b} v\right) \triangleleft_{a} u\right)=\left(\left(w \triangleleft_{a} u\right) \triangleleft_{b} v\right) \circ \theta_{h_{1},\left(b, g_{1}\right),\left(a, f_{1}\right)}, \\
& \left(\beta \beta_{e}\right) \quad \beta_{h,(b, g),(a, f)}^{-1} \circ \beta_{h,(b, g),(a, f)}=\mathbf{1}_{\left(h \triangleleft_{b} g\right) \triangleleft_{b a} f}, \\
& \beta_{h,(b, g),(a, f)} \circ \beta_{h,(b, g),(a, f)}^{-1}=\mathbf{1}_{h \triangleleft_{b}\left(g \triangleleft_{a} f\right)}, \\
& \left(\theta \theta_{e}\right) \quad \theta_{h,(a, f),(b, g)} \circ \theta_{h,(b, g),(a, f)}=\mathbf{1}_{\left(h \triangleleft_{b} g\right) \triangleleft_{a} f}, \\
& \left(\beta \text { pent }_{e}\right)\left(\mathbf{1}_{j} \triangleleft_{c} \beta_{h,(b, g),(a, f)}\right) \circ \beta_{j,\left(c, h \triangleleft_{b} g\right),(a, f)} \circ\left(\beta_{j,(c, h)(b, g)} \triangleleft_{a} \mathbf{1}_{f}\right)= \\
& \beta_{j,(c, h),\left(b, g \triangleleft_{a} f\right)} \circ \beta_{j \triangleleft_{c} h,(b, g),(a, f)}, \\
& \left(\theta \mathrm{YB}_{e}\right) \quad \theta_{j \triangleleft_{a} f,(c, h),(b, g)} \circ\left(\theta_{j,(c, h),(a, f)} \triangleleft_{b} \mathbf{1}_{g}\right) \circ \theta_{j \triangleleft_{c} h,(b, g),(a, f)}= \\
& \left(\theta_{j,(b, g),(a, f)} \triangleleft_{c} \mathbf{1}_{h}\right) \circ \theta_{j \triangleleft_{b} g,(c, h),(a, f)} \circ\left(\theta_{j,(c, h),(b, g)} \triangleleft_{a} \mathbf{1}_{f}\right), \\
& \left(\beta \theta 1_{e}\right) \quad\left(\mathbf{1}_{j} \triangleleft_{c} \theta_{h,(b, g),(a, f)}\right) \circ \beta_{j,\left(c, h \triangleleft_{b} g\right),(a, f)} \circ\left(\beta_{j,(c, h),(b, g)} \triangleleft_{a} \mathbf{1}_{f}\right)= \\
& \beta_{j,\left(c, h \triangleleft_{a} f\right),(b, g)} \circ\left(\beta_{j,(c, h),(a, f)} \triangleleft_{b} \mathbf{1}_{g}\right) \circ \theta_{j \triangleleft_{c} h,(b, g),(a, f)}, \\
& \left(\beta \theta 2_{e}\right) \quad \theta_{j,\left(c, h \triangleleft_{b} g\right),(a, f)} \circ\left(\beta_{j,(c, h),(b, g)} \triangleleft_{a} \mathbf{1}_{f}\right)= \\
& \beta_{j \triangleleft_{a} f,(c, h),(b, g)} \circ\left(\theta_{j,(c, h),(a, f)} \triangleleft_{b} \mathbf{1}_{g}\right) \circ \theta_{j \triangleleft_{c} h,(b, g),(a, f)} .
\end{aligned}
$$


As rules we have symmetry and transitivity of $=$, and the congruence rules for $\circ$ and $\triangleleft_{a}$ (just put $\circ$ and $\triangleleft_{a}$ for $\xi$ in the schema at the end of Section 7). This concludes our definition of the equations of $\mathcal{W O}_{e}^{-}$, and of the category $\mathcal{W O}_{e}^{-}$.

\section{The CATEgory $\mathcal{W} \mathcal{O}_{e}$}

We add now to $\mathcal{W O}_{e}^{-}$what is needed to obtain the unitary category $\mathcal{W O}_{e}$.

The objects of $\mathcal{W O}_{e}$ are the terms of $\mathcal{O}_{e}$ (see Section 3). The arrow terms of $\mathcal{W} \mathcal{O}_{e}$ are defined like those of $\mathcal{W O}_{e}^{-}$in the preceding section with the following additional basic arrow terms, derived from the equations $\left(\right.$ unit $\left._{e}\right)$ of Section 3:

$$
\begin{array}{ll}
\mu_{f, a}: f \triangleleft_{a} \mathbf{I} \rightarrow f, & \mu_{f, a}^{-1}: f \rightarrow f \triangleleft_{a} \mathbf{I}, \\
\lambda_{f}: \mathbf{I} \triangleleft_{e} f \rightarrow f, & \lambda_{f}^{-1}: f \rightarrow \mathbf{I} \triangleleft_{e} f .
\end{array}
$$

The equation of $\mathcal{W O}_{e}$ between arrow terms are defined like those of $\mathcal{W O}_{e}^{-}$in the preceding section with the following additional axiomatic equations:

$\left(\mu\right.$ nat $\left._{e}\right) \quad \mu_{f_{2}, a} \circ\left(u \triangleleft_{a} \mathbf{1}_{\mathbf{I}}\right)=u \circ \mu_{f_{1}, a}$,

$\left(\lambda\right.$ nat $\left._{e}\right) \quad \lambda_{f_{2}} \circ\left(\mathbf{1}_{\mathbf{I}} \triangleleft_{e} u\right)=u \circ \lambda_{f_{1}}$,

$\left(\mu \mu_{e}\right) \quad \mu_{f, a}^{-1} \circ \mu_{f, a}=\mathbf{1}_{f \triangleleft_{a} \mathbf{I}}, \quad \mu_{f, a} \circ \mu_{f, a}^{-1}=\mathbf{1}_{f}$,

$\left(\lambda \lambda_{e}\right) \quad \lambda_{f}^{-1} \circ \lambda_{f}=\mathbf{1}_{\mathbf{I} \triangleleft_{e} f}, \quad \lambda_{f} \circ \lambda_{f}^{-1}=\mathbf{1}_{f}$,

$\left(\beta \mu \lambda_{e}\right) \quad \beta_{h,(b, \mathbf{I}),(e, f)}=\left(\mathbf{1}_{h} \triangleleft_{b} \lambda_{f}^{-1}\right) \circ\left(\mu_{h, b} \triangleleft_{b} \mathbf{1}_{f}\right)$,

$\left(\theta \mu_{e}\right) \quad \theta_{h,(b, \mathbf{I}),(a, f)}=\mu_{h \triangleleft_{a} f, b}^{-1} \circ\left(\mu_{h, b} \triangleleft_{a} \mathbf{1}_{f}\right)$.

\section{1. $\mathcal{W} \mathcal{O}_{e}$ AND $\mathcal{W O}_{u}$}

In this section we establish the relationship between $\mathcal{W} \mathcal{O}_{e}$ and $\mathcal{W} \mathcal{O}_{u}$. We show that $\mathcal{W} \mathcal{O}_{u}$ is the disjoint union of isomorphic copies of $\mathcal{W O}_{e}$.

For every $\mathbf{N}^{+}$-word $a$ we define inductively a map $a$. from the arrow terms of $\mathcal{W O}_{u}$ to the arrow terms of $\mathcal{W O}_{u}$ :

$$
\begin{aligned}
& a \cdot \nu_{f}=\nu_{a \cdot f}, \quad \text { where } \nu \text { is } \mathbf{1}, \lambda \text { and } \lambda^{-1}, \\
& a \cdot \nu_{f, b}=\nu_{a \cdot f, a b}, \quad \text { where } \nu \text { is } \mu \text { and } \mu^{-1}, \\
& a \cdot \zeta_{h, g, f}=\zeta_{a \cdot h, a \cdot g, a \cdot f}, \quad \text { where } \zeta \text { is } \beta, \beta^{-1} \text { and } \theta, \\
& a \cdot(v \xi u)=(a \cdot v) \xi(a \cdot u), \quad \text { where } \xi \text { is } \circ \text { and } \triangleleft .
\end{aligned}
$$

The definition of $a$. on the objects of $\mathcal{W} \mathcal{O}_{u}$, i.e. the terms of $\mathcal{O}_{u}$, which is mentioned in the indices above, is given in Section 6. It is clear that $a$. induces an endofunctor of $\mathcal{W O}_{u}$, since $u=v$ in $\mathcal{W O}_{u}$ clearly implies $a \cdot u=a \cdot v$ in $\mathcal{W O}_{u}$.

We need $a$. to define inductively a map $U$ from the arrow terms of $\mathcal{W O} \mathcal{O}_{e}$ to the arrow terms of $\mathcal{W O}_{u}$ :

$$
\begin{aligned}
& U\left(\nu_{f}\right)=\nu_{U(f)}, \quad \text { where } \nu \text { is } \mathbf{1}, \lambda \text { and } \lambda^{-1}, \\
& U\left(\nu_{f, a}\right)=\nu_{U(f), a}, \quad \text { where } \nu \text { is } \mu \text { and } \mu^{-1}, \\
& U\left(\zeta_{h,(b, g),(a, f)}\right)=\zeta_{U(h), b \cdot U(g), b a \cdot U(f)}, \quad \text { where } \zeta \text { is } \beta \text { and } \beta^{-1}, \\
& U\left(\theta_{h,(b, g),(a, f)}\right)=\theta_{U(h), b \cdot U(g), a \cdot U(f)},
\end{aligned}
$$




$$
\begin{aligned}
& U(v \circ u)=U(v) \circ U(u), \\
& U\left(v \triangleleft_{a} u\right)=U(v) \triangleleft a \cdot U(u) .
\end{aligned}
$$

The map $U$ mentioned in the indices of this definition is the map $U$ defined in Section 6 .

Next we define inductively a map $E$ from the arrow terms of $\mathcal{W} \mathcal{O}_{u}$ to the arrow terms of $\mathcal{W O}_{e}$ :

$$
\begin{aligned}
& E\left(\nu_{f}\right)=\nu_{E(f)}, \quad \text { where } \nu \text { is } \mathbf{1}, \lambda \text { and } \lambda^{-1}, \\
& E\left(\nu_{f, a}\right)=\nu_{E(f), a}, \quad \text { where } \nu \text { is } \mu \text { and } \mu^{-1}, \\
& E\left(\zeta_{h, g, f}\right)=\zeta_{E(h),(t(h) \backslash t(g), E(g)),(t(g) \backslash t(f), E(f))}, \quad \text { where } \zeta \text { is } \beta \text { and } \beta^{-1}, \\
& E\left(\theta_{h, g, f}\right)=\theta_{E(h),(t(h) \backslash t(g), E(g)),(t(h) \backslash t(f), E(f))}, \\
& E(v \circ u)=E(v) \circ E(u), \\
& E(v \triangleleft u)=E(v) \triangleleft_{t(g) \backslash t(f)} E(u), \text { for } u: f \rightarrow f^{\prime} \text { and } v: g \rightarrow g^{\prime} .
\end{aligned}
$$

The map $E$ mentioned in the indices of this definition is the map $E$ defined in Section 6 .

With the help of (st a.) and Lemma 6.4 of Section 6, it is easy to establish the following by induction on the complexity of $u$.

Lemma 11.1. For every arrow term $u$ of $\mathcal{W O}_{u}$ we have that $E(a \cdot u)$ is $E(u)$.

This lemma serves for the first of the following two propositions, which are proved by inductions on the complexity of $u$.

Proposition 11.2. For every arrow term $u$ of $\mathcal{W O}_{e}$ we have that $E(U(u))$ is $u$.

Proposition 11.3. For every arrow term $u: f \rightarrow f^{\prime}$ of $\mathcal{W O}_{u}$ we have that $t(f) \cdot U(E(u))$ is $u$.

In the proofs of these propositions we rely on Propositions 6.5 and 6.6. As an example, which is perhaps not quite straightforward, we give the following case in the proof of Proposition 11.3 .

$$
\begin{aligned}
& t(h) \cdot U\left(E\left(\beta_{h, g, f}\right)\right)=t(h) \cdot U\left(\beta_{E(h),(t(h) \backslash t(g), E(g)),(t(g) \backslash t(f), E(f))}\right) \\
& =\beta_{t(h) \cdot U(E(h)), t(h)(t(h) \backslash t(g)) \cdot U(E(g)), t(h)(t(h) \backslash t(g))(t(g) \backslash t(f)) \cdot U(E(f))} \\
& =\beta_{t(h) \cdot U(E(h)), t(g) \cdot U(E(g)), t(f) \cdot U(E(f))}=\beta_{h, g, f} \text {, by Proposition 6.6. }
\end{aligned}
$$

Next we establish the following two propositions by inductions on the length of derivation, where the main burden is in the bases of the inductions, with axiomatic equations.

Proposition 11.4. If $u=v$ in $\mathcal{W O}_{e}$, then $U(u)=U(v)$ in $\mathcal{W O}_{u}$.

Proposition 11.5. If $u=v$ in $\mathcal{W O}_{u}$, then $E(u)=E(v)$ in $\mathcal{W} \mathcal{O}_{e}$.

The inductive proofs of these two propositions, which are lengthy but straightforward, yield more than what is stated in the propositions. Every derivation in the equational system of $\mathcal{W O} \mathcal{O}_{e}$ is translated into a derivation in the equational system of $\mathcal{W} \mathcal{O}_{u}$ in a "homomorphic" manner, and vice versa. This means, for example, that $\left(\beta \theta 1_{e}\right)$ goes into $(\beta \theta 1)$, etc. These two propositions yield that $U$ induces a functor from $\mathcal{W} \mathcal{O}_{e}$ to $\mathcal{W} \mathcal{O}_{u}$, and $E$ a functor in the opposite direction.

Let $\mathcal{W O}_{e}(X)$ be the full subcategory of $\mathcal{W} \mathcal{O}_{e}$ whose objects are all the terms $f$ of $\mathcal{O}_{e}$ such that $s(f)=X$. The category $\mathcal{W} \mathcal{O}_{e}$ is the disjoint union of its subcategories $\mathcal{W O}_{e}(X)$ for all the nominal arities $X$. 
Let $\mathcal{W} \mathcal{O}_{u}(X, b)$ be the full subcategory of $\mathcal{W} \mathcal{O}_{u}$ whose objects are all the terms $f$ of $\mathcal{O}_{u}$ such that $s(f)=X$ and $t(f)=b$. Let $\mathcal{W} \mathcal{O}_{u}(b)$ be the union of the categories $\mathcal{W} \mathcal{O}_{u}(X, b)$ for all the nominal arities $X$ (this union is disjoint). The category $\mathcal{W} \mathcal{O}_{u}$ is the disjoint union of its subcategories $\mathcal{W O}_{u}(b)$ for all $\mathbf{N}^{+}$-words $b$. We can prove the following.

Proposition 11.6. The categories $\mathcal{W O}_{e}$ and $\mathcal{W} \mathcal{O}_{u}(e)$ are isomorphic.

Proof. Propositions 11.2,11.5 show that, on the one hand, the functor $U$ and, on the other hand, the functor $E$ restricted to $\mathcal{W O}_{u}(e)$ are inverse to each other.

More precisely, we have that $\mathcal{W} \mathcal{O}_{e}(X)$ and $\mathcal{W O}_{u}(X, e)$ are isomorphic categories. For that we just restrict further the functors $U$ and $E$.

Let $\mathcal{W} \mathcal{O}_{u}(b \bullet)$ be the union of the categories $\mathcal{W O}_{u}(b a)$ for all $\mathbf{N}^{+}$-words $a$. We define a functor $b \backslash$ from $\mathcal{W O}_{u}(b \bullet)$ to $\mathcal{W} \mathcal{O}_{u}$ by stipulating that $b \backslash u$ is $u$ with every index $b a$ replaced by $a$; moreover, $b \backslash(v \xi u)$ is $(b \backslash v) \xi(b \backslash u)$ for $\xi$ being $\circ$ and $\triangleleft$ (cf. the definition of the functor $a$. at the beginning of the section). We can prove the following.

Proposition 11.7. For every $\mathbf{N}^{+}$-word $b$ the categories $\mathcal{W O}_{u}$ and $\mathcal{W O}_{u}(b \bullet)$ are isomorphic.

Proof. From $\mathcal{W O}_{u}$ and $\mathcal{W O}_{u}(b \bullet)$ we have the functor $b$, and it is easy to see that the functor $b \backslash$ is its inverse.

We can also prove the following.

Proposition 11.8. For all $\mathbf{N}^{+}$-words $a$ and $b$ the categories $\mathcal{W O}_{u}(a)$ and $\mathcal{W O}_{u}(b a)$ are isomorphic.

Proof. We restrict the functors $b$. and $b \backslash$ to the subcategory $\mathcal{W} \mathcal{O}_{u}(a)$ of $\mathcal{W} \mathcal{O}_{u}$ and the subcategory $\mathcal{W O}_{u}(b a)$ of $\mathcal{W O}_{u}(b \bullet)$.

As a corollary of this proposition, we obtain that for every $\mathbf{N}^{+}$-word $b$ the categories $\mathcal{W} \mathcal{O}_{u}(e)$ and $\mathcal{W} \mathcal{O}_{u}(b)$ are isomorphic, and hence, with Proposition 11.6, we have that $\mathcal{W} \mathcal{O}_{e}$ and $\mathcal{W O}_{u}(b)$ are isomorphic. Since the category $\mathcal{W O}_{u}$ is the disjoint union of the categories $\mathcal{W} \mathcal{O}_{u}(b)$ for all $\mathbf{N}^{+}$-words $b$, we may conclude that $\mathcal{W} \mathcal{O}_{u}$ is the disjoint union of isomorphic copies of $\mathcal{W} \mathcal{O}_{e}$.

Propositions 6.11, 6.12 and 6.13 are parallel to Propositions 11.6, 11.7 and 11.8. The propositions of Section 6 deal with algebraic structures with partial operations satisfying various equations related to (unit), (assoc 1) and (assoc 2), while the categories $\mathcal{W O}_{e}$ and $\mathcal{W} \mathcal{O}_{u}$ do not satisfy these equations, but have arrows that are isomorphisms instead of them. Nevertheless, the former propositions indicate how the functors of this section do not preserve only $\mathbf{1}$ and $\circ$, but also insertion, as a partial operation both on objects and on arrows. What is preserved is either the whole family of insertion operations indexed with $\mathbf{N}^{+}$-words, or just the single partial operation $\triangleleft$ (which corresponds to $\triangleleft_{e}$ ). What is preserved is also $\mathbf{I}$, or objects derived from it.

Everything we said in this section about the relationship between $\mathcal{W} \mathcal{O}_{e}$ and $\mathcal{W O}_{u}$ can be restricted to the non-unitary categories $\mathcal{W O}_{e}^{-}$and $\mathcal{W O}_{u}^{-}$. 


\section{Operads, Cat-operads and weak Cat-operads}

DEFINITION OF OPERAD. The standard general notion of operad with insertions primitive would be based on the operad $\mathcal{O}$ of Section 2. It defines a class of partial algebras in which $\mathcal{O}$ is freely generated by $G$ and $\alpha_{G}$. These algebras have a carrier $C$ made of elements called operadic operations, such that for every operadic operation $\varphi$ of $C$ we have an arity in $\mathbf{N}$, and they have the family of insertions $\left\{\triangleleft_{k} \mid k \in \mathbf{N}^{+}\right\}$, which are partial operations on operadic operations, and $\mathbf{I}$ with arity 1 , for which equations like those of $\mathcal{O}$ hold. The notion of multicategory is a generalization of this notion where an arity $n$ is replaced by a sequence of $n$ occurrences of some objects, and moreover we have an object as a target (see [Lam89]). An operad is a one-object multicategory.

First definition of CAT-Operad. A Cat-operad is an operad that in addition has arrows between operadic operations of the same arity. We have an identity arrow for every operadic operation, and the arrows are closed under the partial operations of composition and insertions, which are now not only partial operations on operadic operations, but also partial operations on arrows. This structure is a category, i.e. identity arrows and composition satisfy the categorial equations (see Section 7). Since the enrichment is over Cat, the category of all small categories, it is assumed that the hom-categories of this category are small, but this is not an essential matter. We have moreover in this category the equations (ins $1_{e}$ ) and $\left(\right.$ ins $2_{e}$ ) of Section 9 with $a$ replaced by $n$, and the analogues of the equations (unit), (assoc 1) and (assoc 2) for arrows (see Section 2). In these analogues $\mathbf{I}$ is replaced by $\mathbf{1}_{\mathbf{I}}$ and $\varphi, \gamma$ and $\chi$ are replaced by variables for arrows. If $u$ is such a variable, then $\alpha(u)$ is the arity of the source or target of $u$, which must have the same arity.

SECond Definition of CAT-Operad. In other words, a Cat-operad can be defined as the disjoint union of categories $\mathcal{C}_{k}$; in $\mathcal{C}_{k}$ all the objects, called operadic operations, have arity $k$. In $\mathcal{C}_{1}$ we have a special object $\mathbf{I}$. We have moreover the bifunctors $\triangleleft_{n}: \mathcal{C}_{k} \times \mathcal{C}_{l} \rightarrow \mathcal{C}_{k-1+l}$, for $1 \leq n \leq k$, which satisfy the equations (unit), (assoc 1) and (assoc 2), and analogous equations for arrows.

We base our general notion of weak Cat-operad on $\mathcal{W} \mathcal{O}_{e}$. Let $\left\langle G, \alpha_{G}, \mathcal{G}\right\rangle$ be a triple (not in the sense of monad), where $G$ is a set, $\alpha_{G}$ is a function from $G$ to $\mathbf{N}$ and $\mathcal{G}$ is a

directed graph $G \stackrel{\text { dom }}{\longleftarrow} A \stackrel{\text { cod }}{\longrightarrow} G$ (in the sense of MLa98, Section I.2) such that for every $u$ in $A$ we have

$$
\alpha_{G}(\operatorname{dom}(u))=\alpha_{G}(\operatorname{cod}(u)) .
$$

Let $X$ be a category whose objects are such triples and whose arrows from $\left\langle G, \alpha_{G}, \mathcal{G}\right\rangle$ to $\left\langle G^{\prime}, \alpha_{G^{\prime}}, \mathcal{G}^{\prime}\right\rangle$ are graph morphisms $\varphi: \mathcal{G} \rightarrow \mathcal{G}^{\prime}$ such that for every $x$ in $G$ we have that $\alpha_{G}(x)=\alpha_{G^{\prime}}(\varphi(x))$.

We define the category $\mathcal{W} \mathcal{O}_{e}\left\langle G, \alpha_{G}, \mathcal{G}\right\rangle$ as we have defined $\mathcal{W} \mathcal{O}_{e}$ in Sections 9-10 based on $G$ and $\alpha_{G}$ save that in the definition of arrow terms we add the clause

if $u: x \rightarrow y$ is in $A$, then it is an arrow term.

We say that a functor $F: \mathcal{W} \mathcal{O}_{e}\left\langle G, \alpha_{G}, \mathcal{G}\right\rangle \rightarrow \mathcal{W} \mathcal{O}_{e}\left\langle G^{\prime}, \alpha_{G^{\prime}}, \mathcal{G}^{\prime}\right\rangle$ preserves the $\mathcal{W} \mathcal{O}_{e}$ structure when

for objects:

$$
F(\mathbf{I})=\mathbf{I}
$$




$$
F\left(g \triangleleft_{a} f\right)=F g \triangleleft_{a^{\prime}} F f, \text { where } K_{s(g)}(a)=K_{s(F g)}\left(a^{\prime}\right) ;
$$

and for arrows:

$$
\begin{array}{r}
F\left(\beta_{h,(b, g),(a, f)}\right)=\beta_{F h,\left(b^{\prime}, F g\right),\left(a^{\prime}, F f\right)}, \text { where } K_{s(g)}(a)=K_{s(F g)}\left(a^{\prime}\right) \text { and } \\
K_{s(h)}(b)=K_{s(F h)}\left(b^{\prime}\right) ;
\end{array}
$$

similarly for $\beta^{-1}, \theta, \mu, \mu^{-1}, \lambda$ and $\lambda^{-1}$;

$F\left(v \triangleleft_{a} u\right)=F v \triangleleft_{a^{\prime}} F u$, where $u: f \rightarrow f^{\prime}, v: g \rightarrow g^{\prime}$ and

$$
K_{s(g)}(a)=K_{s(F g)}\left(a^{\prime}\right)
$$

(see Section 4 for the definition of $K$ ).

Let $Y$ be the category whose objects are all the categories $\mathcal{W} \mathcal{O}_{e}\left\langle G, \alpha_{G}, \mathcal{G}\right\rangle$ and whose arrows are all the functors that preserve the $\mathcal{W} \mathcal{O}_{e}$ structure. Every arrow $\varphi:\left\langle G, \alpha_{G}, \mathcal{G}\right\rangle \rightarrow$ $\left\langle G^{\prime}, \alpha_{G^{\prime}}, \mathcal{G}^{\prime}\right\rangle$ of $X$ induces a function $\varphi_{0}$ from the objects of $\mathcal{W} \mathcal{O}_{e}\left\langle G, \alpha_{G}, \mathcal{G}\right\rangle$ to the objects of $\mathcal{W} \mathcal{O}_{e}\left\langle G^{\prime}, \alpha_{G^{\prime}}, \mathcal{G}^{\prime}\right\rangle$ and a function $\varphi_{1}$ from the arrow terms of $\mathcal{W} \mathcal{O}_{e}\left\langle G, \alpha_{G}, \mathcal{G}\right\rangle$ to the arrow terms of $\mathcal{W} \mathcal{O}_{e}\left\langle G^{\prime}, \alpha_{G^{\prime}}, \mathcal{G}^{\prime}\right\rangle$ in a natural way. It is straightforward to verify that for the arrow terms $u$ and $v$, if $u=v$ in $\mathcal{W} \mathcal{O}_{e}\left\langle G, \alpha_{G}, \mathcal{G}\right\rangle$, then $\varphi_{1}(u)=\varphi_{1}(v)$ in $\mathcal{W} \mathcal{O}_{e}\left\langle G^{\prime}, \alpha_{G^{\prime}}, \mathcal{G}^{\prime}\right\rangle$. Hence the functions $\varphi_{0}$ and $\varphi_{1}$ underlie a functor $L \varphi$ from $\mathcal{W} \mathcal{O}_{e}\left\langle G, \alpha_{G}, \mathcal{G}\right\rangle$ to $\mathcal{W} \mathcal{O}_{e}\left\langle G^{\prime}, \alpha_{G^{\prime}}, \mathcal{G}^{\prime}\right\rangle$. It is not difficult to check that $L \varphi$ preserves the $\mathcal{W} \mathcal{O}_{e}$ structure.

Hence we have a functor $L: X \rightarrow Y$ which maps $\left\langle G, \alpha_{G}, \mathcal{G}\right\rangle$ to $\mathcal{W} \mathcal{O}_{e}\left\langle G, \alpha_{G}, \mathcal{G}\right\rangle$ and $\varphi$ to $L \varphi$. On the other hand, we can define the forgetful functor $R: Y \rightarrow X$ so that for $C$ being $\mathcal{W} \mathcal{O}_{e}\left\langle G, \alpha_{G}, \mathcal{G}\right\rangle$ we have that $R(C)=\left\langle H, \alpha_{H}, \mathcal{H}\right\rangle$, where

$H=O b(C)$, i.e. the set of terms of $\mathcal{O}_{e}$ based on $G$ and $\alpha_{G}$,

$\alpha_{H}(f)=|s(f)|$, and

$\mathcal{H}$ is the directed graph underlying the category $C$.

Proposition 12.1. The functor $L$ is the left adjoint of $R$.

Proof. The proof that $Y\left(\mathcal{W} \mathcal{O}_{e}\left\langle G, \alpha_{G}, \mathcal{G}\right\rangle, \mathcal{W} \mathcal{O}_{e}\left\langle G^{\prime}, \alpha_{G^{\prime}}, \mathcal{G}^{\prime}\right\rangle\right)$ is naturally isomorphic to $X\left(\left\langle G, \alpha_{G}, \mathcal{G}\right\rangle, R\left(\mathcal{W} \mathcal{O}_{e}\left\langle G^{\prime}, \alpha_{G^{\prime}}, \mathcal{G}^{\prime}\right\rangle\right)\right)$ is similar to the proof that $L \varphi$ is a functor that preserves the $\mathcal{W O} \mathcal{O}_{e}$ structure.

Definition of Weak Cat-operad. A weak Cat-operad is an algebra of the monad in $X$ defined by the above adjunction.

Alternatively, a weak Cat-operad can be defined in the style of the second definition of Cat-operad above by copying this definition until we reach the equations (unit), (assoc 1) and (assoc 2). Instead of these equations, we have natural isomorphisms corresponding to $\mu, \lambda, \beta$ and $\theta$ and equations analogous to the equations $\left(\beta \mu \lambda_{e}\right),\left(\theta \mu_{e}\right),\left(\beta\right.$ pent $\left._{e}\right),\left(\theta \mathrm{YB}_{e}\right)$, $\left(\beta \theta 1_{e}\right)$ and $\left(\beta \theta 2_{e}\right)$ of Sections 10 and 9. For example, the equation analogous to $\left(\beta \theta 1_{e}\right)$ would be the following:

$$
\begin{aligned}
\left(\mathbf{1}_{j} \triangleleft_{m} \theta_{h,(l, g),(k-1+\alpha(g), f)}\right) \circ \beta_{j,\left(m, h \triangleleft_{l} g\right),\left(k^{\prime}, f\right)} \circ\left(\beta_{j,(m, h),\left(l^{\prime}, g\right)} \triangleleft_{k^{\prime}} \mathbf{1}_{f}\right)= \\
\beta_{j,\left(m, h \triangleleft_{k} f\right),\left(l^{\prime}, g\right)} \circ\left(\beta_{j,(m, h),(k+m-1, f)} \triangleleft_{l^{\prime}} \mathbf{1}_{g}\right) \circ \theta_{j \triangleleft_{m} h,\left(l^{\prime}, g\right),\left(k^{\prime}, f\right)},
\end{aligned}
$$

where $k^{\prime}=k-1+\alpha(g)+m-1$ and $l^{\prime}=l+m-1$, with $k, l$ and $m$ standing respectively for $K_{s(h)}(a), K_{s(h)}(b)$ and $K_{s(h)}(c)$ (see Section 4 for the definition of $K$ ).

This equation is considerably more complicated than our equation $\left(\beta \theta 1_{e}\right)$, and to eschew such complications is the main reason for introducing our nominal arities. To infer all the equations needed for a full definition of weak Cat-operads, as we inferred the equation above 
from $\left(\beta \theta 1_{e}\right)$, is a lengthy, but straightforward, matter, into which we will not go further in this paper.

\section{3. $\mathcal{W} \mathcal{O}_{e}^{-}$AND HEMIASSOCIAHEDRA}

For particular choices of the nominal arity $X$ the categories $\mathcal{W} \mathcal{O}_{e}^{-}(X)$ defined in Section 11 have an interesting shape. They become representable by polyhedra of a kind analogous to associahedra and permutohedra (see [Sta97], Sta97a], Zie95, Lecture 0, Example 0.10, and GR63 for historical references concerning associahedra and permutohedra). In some cases they are exactly associahedra that involve only $\beta$ arrows, and permutohedra that involve only $\theta$ arrows. These associahedra and permutohedra do not differ from those already considered in the literature in connection with associativity and commutativity isomorphisms of monoidal and symmetric strictly monoidal categories.

It is interesting however to describe associahedra and permutohedra with $\beta$ and $\theta$ arrows mixed. With these two kinds of arrows mixed we obtain also in three dimensions another kind of polyhedron, which was called hemiassociahedron in [DP10] (Example 5.14). That paper investigates in particular the relationship of the hemiassociahedron to the permutohedron (from which, together with [Ton97], one may gather that the hemiassociahedron, conceived as an abstract polytope, can be realized; we will however not go here into this problem, for which one should also consult [DP11]).

In this section we describe nine categories $\mathcal{W O}_{e}^{-}(X)$. The first four may be represented by hemiassociahedra; next we have two that may be represented by three-dimensional associahedra with $\beta$ and $\theta$ mixed, and one that may be represented by a three-dimensional permutohedron with $\beta$ and $\theta$ arrows mixed. Finally we have a category represented by a purely $\beta$ associahedron, and a category represented by a purely $\theta$ permutohedron.

In all our examples, instead of dealing with $\mathcal{W O}_{e}^{-}(X)$ we may deal with the isomorphic category $\mathcal{W O}_{u}^{-}(X, e)$. In our first example, which is given with more details, we concentrate on this category, simpler to deal with. In other examples, with less details, we would proceed analogously.

Example 13.1. Let $G=\{x\}$ and let $\alpha_{G}(x)=2$. Let $X=\{111,112,121,122,21,22\}$. Every object of $\mathcal{W O}_{u}^{-}(X, e)$, which is a term $f$ of $\mathcal{O}_{u}^{-}$with $s(f)=X$ and $t(f)=e$, records a destruction of the following binary tree, whose leaves make $X$ :

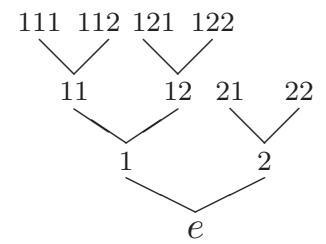

For example, the object $f$, which is

$$
(e \cdot x \triangleleft 2 \cdot x) \triangleleft((1 \cdot x \triangleleft 11 \cdot x) \triangleleft 12 \cdot x),
$$

records the following destruction. The main insertion $\triangleleft$ of our object is first removed. This leaves us with the $\mathcal{O}_{u}^{-}$terms $f_{1}$ and $f_{2}$, which are respectively

$$
(1 \cdot x \triangleleft 11 \cdot x) \triangleleft 12 \cdot x \quad \text { and } \quad e \cdot x \triangleleft 2 \cdot x,
$$

and which record destructions of the following two trees: 

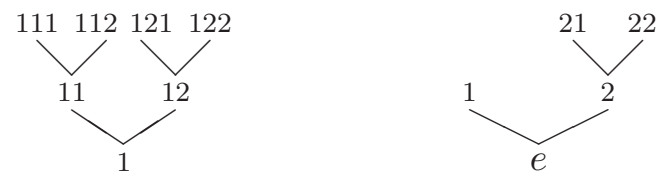

We have $t\left(f_{1}\right)=1 \in s\left(f_{2}\right)$.

Next we concentrate on the destruction recorded by $f_{1}$. We remove its main insertion, and next the insertion remaining on its left, which leaves three residual trees:

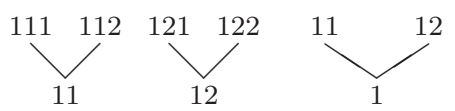

This destruction of $f_{1}$ may be recorded also by $(12) \cdot(11)$, which indicate that we broke the tree of $f_{1}$ first at the vertex 12 , and next broke one of the remaining trees at the vertex 11 .

The destruction of $f_{2}$ proceeds by breaking its tree at the vertex 2 , which leaves the two trees

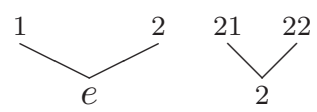

This destruction may also be recorded by 2, the vertex where the tree broke.

The destruction of $f_{1}$ and $f_{2}$ may have gone simultaneously, and we indicate that by writing $((12) \cdot(11))+2$, which is equal to $2+((12) \cdot(11))$. On the other hand, $\cdot$ is not commutative, and indicates successive steps of destruction. Both $\cdot$ and + are on the other hand associative. Our complete destruction of $f$ may then be recorded by $1 \cdot(((12) \cdot(11))+2)$. (Every destruction is, of course, a construction in reverse order.)

Consider terms built out as in this example out of the names of the inner vertices of a tree with the help of the operations - and + . Some of these terms are in one-to-one correspondence with the terms of $\mathcal{O}_{u}^{-}$in our examples, so that the associative and noncommutative operation - corresponds to successive steps of destruction, while the associative and commutative operation + corresponds to simultaneous steps of destruction. These terms are shorter than the terms of $\mathcal{O}_{u}^{-}$.

This matter is treated more formally in [DP10, where such terms with · and + are called S-trees. (The one-to-one correspondence mentioned in the preceding paragraph exists because the value of $\alpha_{G}$ is greater than or equal to 2 , and $\alpha_{G}$ happens to be one-one.) To shorten these terms further we will write $a, b, c$ and $d$ for respectively $11,12,1$ and 2 . The $\mathbf{N}^{+}$-words $a, b, c$ and $d$ stand for the inner vertices of the tree of $f$, i.e. vertices that are neither leaves nor roots. These vertices in this tree make the following graph:

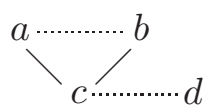

where the solid edge $\{c, a\}$ indicates that the vertices $c$ and $a$ are immediately one above the other in the tree; and analogously for the solid edge $\{c, b\}$; the dotted edge $\{a, b\}$ indicates that $a$ and $b$ are vertices growing out of the same predecessor, and analogously with $\{c, d\}$.

The destruction of the tree of $f$ may be understood as the destruction of the graph we have just given, because in destructing the tree we break it at inner vertices. The destruction of the graph is based on vertex removal (which one finds in Ulam's conjecture; see [Har69], Chapter 2). More details and a general theory concerning the destruction of graphs may be found in [DP10]. 
The S-tree $c \cdot((b \cdot a)+d)$, which we had above, and which corresponds to our object $f$ of $\mathcal{W O}_{u}^{-}(X, e)$, and all the other S-trees, which correspond bijectively to all the other objects of $\mathcal{W O}_{u}^{-}(X, e)$, make the vertices of the following hemiassociahedron:

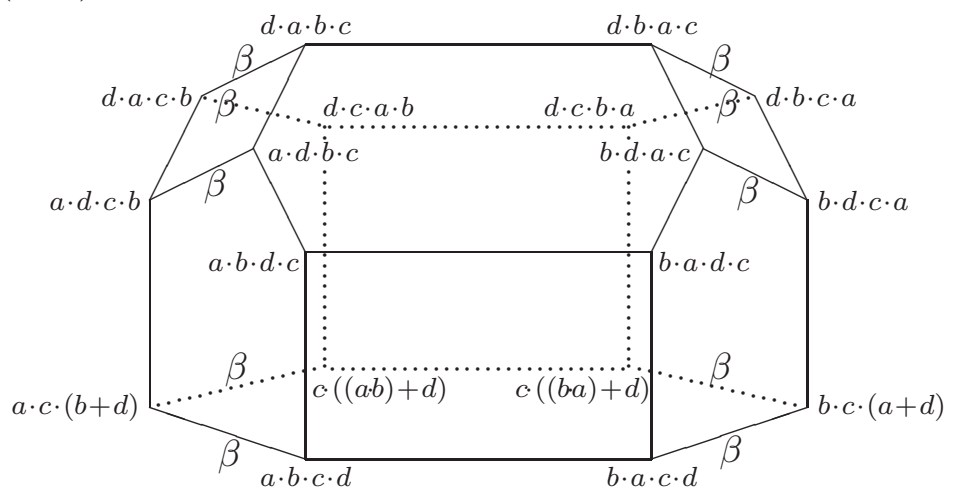

The edges with the label $\beta$ stand for $\beta$ arrows, i.e. arrows built with $\mathbf{1}, \triangleleft$ and one occurrence of $\beta$ (or $\beta^{-1}$ ), while the remaining edges stand for $\theta$ arrows, i.e. arrows built with $\mathbf{1}, \triangleleft$ and one occurrence of $\theta$.

In this hemiassociahedron, the two hexagonal faces with both $\beta$ and $\theta$ arrows (actually, each with four $\beta$ arrows and two $\theta$ arrows) stand for the commuting diagram corresponding to the equation $(\beta \theta 1)$, or $\left(\beta \theta 1_{e}\right)$ if we are in $\mathcal{W O}_{e}^{-}(X)$. The remaining hexagonal face, which has only $\theta$ arrows, is analogously related to the equation $(\theta \mathrm{YB})$, or $\left(\theta \mathrm{YB}_{e}\right)$. The four pentagonal faces are all of the $\beta$ and $\theta$ mixed type (each with two $\beta$ arrows and three $\theta$ arrows), and they are related to the equation $(\beta \theta 2)$, or $\left(\beta \theta 2_{e}\right)$. The four square faces are related to the equation $(\theta$ nat $)$, or $\left(\theta\right.$ nat $\left._{e}\right)$.

This hemiassociahedron shows that the particular instance of $(\theta \mathrm{YB})$ that corresponds to one of the hexagonal faces is derivable from the equations corresponding to the other faces. However, not all instances of $(\theta$ YB) are derivable in this manner (cf. Examples 13.4 and 13.9). The same applies to the equation $\left(\theta \mathrm{YB}_{e}\right)$.

In the remaining examples we will not make comments so detailed as here. It is however easy to recognize in the pictures of other hemiassociahedra, and of associahedra and permutohedra, the equations related to the two-dimensional faces. Besides equations we had in this example, we will encounter also $(\beta$ pent) and $(\beta$ nat $)$, which appear already in the next example.

Example 13.2. Let $G$ and $\alpha_{G}$ be as in the preceding example. Let $X=\{1111,1112,112$, $121,122,2\}$. Let $a, b, c$ and $d$ stand respectively for $12,1,11$ and 111 , which are the inner vertices of the following binary tree, whose leaves make $X$ :
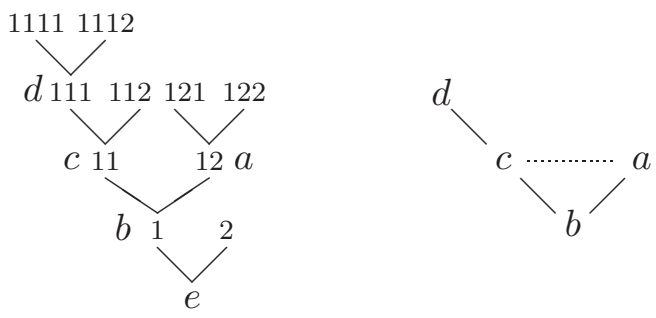

On the right of our tree one finds the graph of the inner vertices.

The objects of $\mathcal{W O}_{e}^{-}(X)$ correspond bijectively to the vertices of the following hemiassociahedron: 


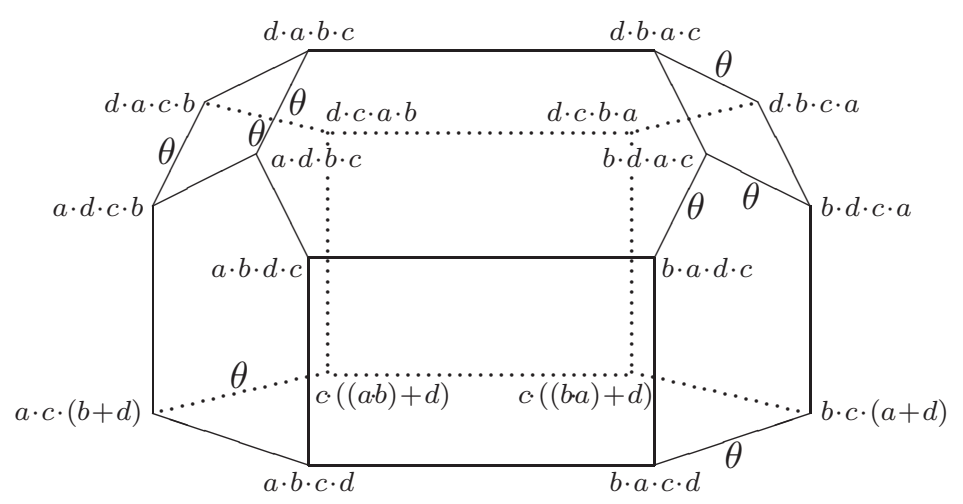

whose edges with $\theta$ stand for $\theta$ arrows, while the remaining edges stand for $\beta$ arrows.

Example 13.3. Let $G$ and $\alpha_{G}$ be as in the preceding two examples. Let $X=\{1111,1112$, $1121,1122,12,2\}$. Let $a, b, c$ and $d$ stand respectively for $111,112,11$ and 1 , which are the inner vertices of the following binary tree, whose leaves make $X$ :

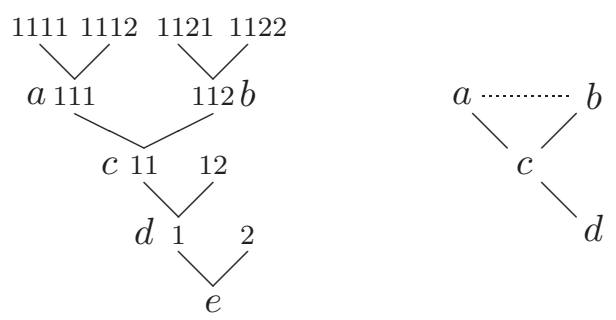

On the right of our tree one finds the graph of the inner vertices.

The objects of $\mathcal{W O}_{e}^{-}(X)$ correspond bijectively to the vertices of the following hemiassociahedron:

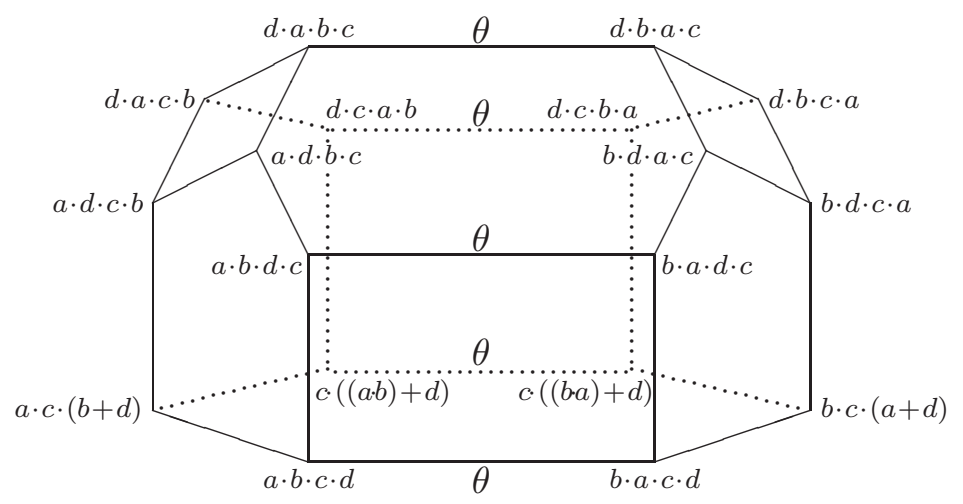

whose edges with $\theta$ stand for $\theta$ arrows, while the remaining edges stand for $\beta$ arrows.

Example 13.4. Let $G=\{x, y\}$, and let $\alpha_{G}(x)=2$ and $\alpha_{G}(y)=3$. Let $X=\{111,112,12$, $21,22,31,32\}$. Let $a, b, c$ and $d$ stand respectively for $3,2,1$ and 11 , which are the inner vertices of the following tree, whose leaves make $X$ : 

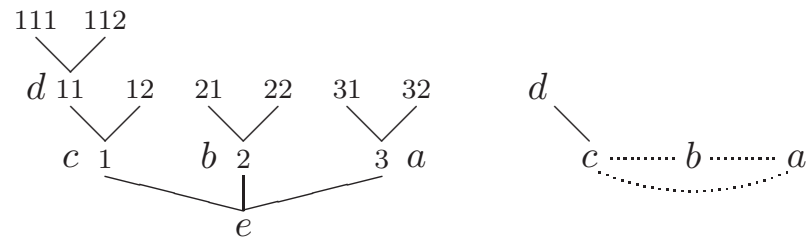

On the right of our tree one finds the graph of the inner vertices.

The objects of $\mathcal{W O}_{e}^{-}(X)$ correspond bijectively to the vertices of the following hemiassociahedron:

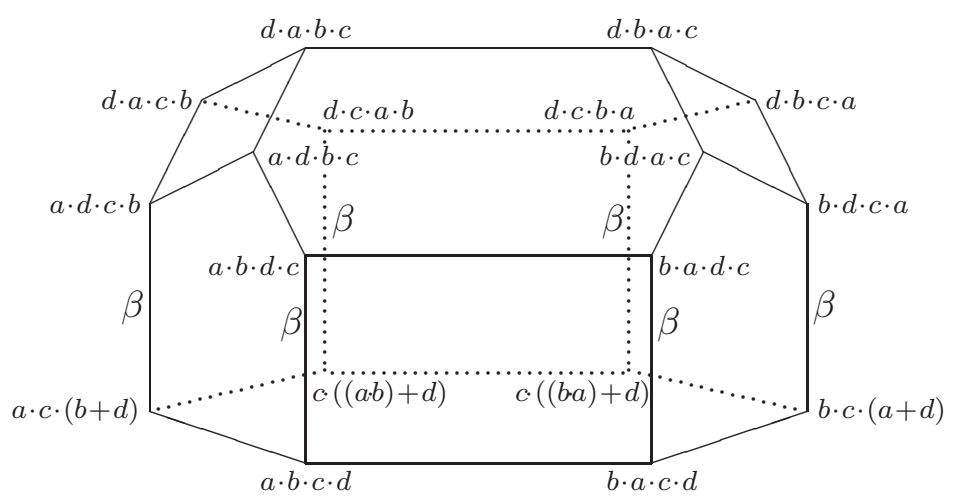

whose edges with $\beta$ stand for $\beta$ arrows, while the remaining edges stand for $\theta$ arrows.

Example 13.5. Let $G$ and $\alpha_{G}$ be as in Examples 13.1 13.3. Let $X=\{1111,1112,112,12$, $21,22\}$. Let $a, b, c$ and $d$ stand respectively for $2,1,11$ and 111 , which are the inner vertices of the following binary tree, whose leaves make $X$ :
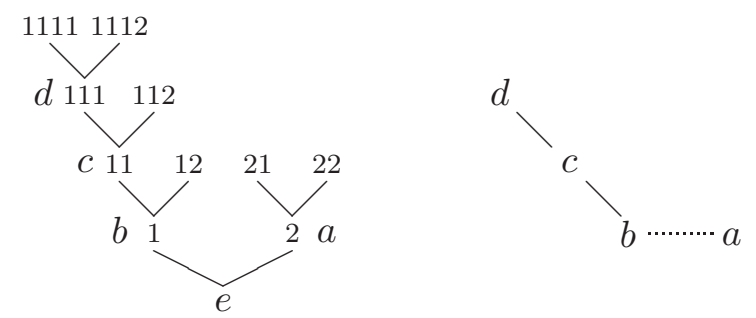

On the right of our tree one finds the graph of the inner vertices.

The objects of $\mathcal{W O}_{e}^{-}(X)$ correspond bijectively to the vertices of the following threedimensional associahedron:

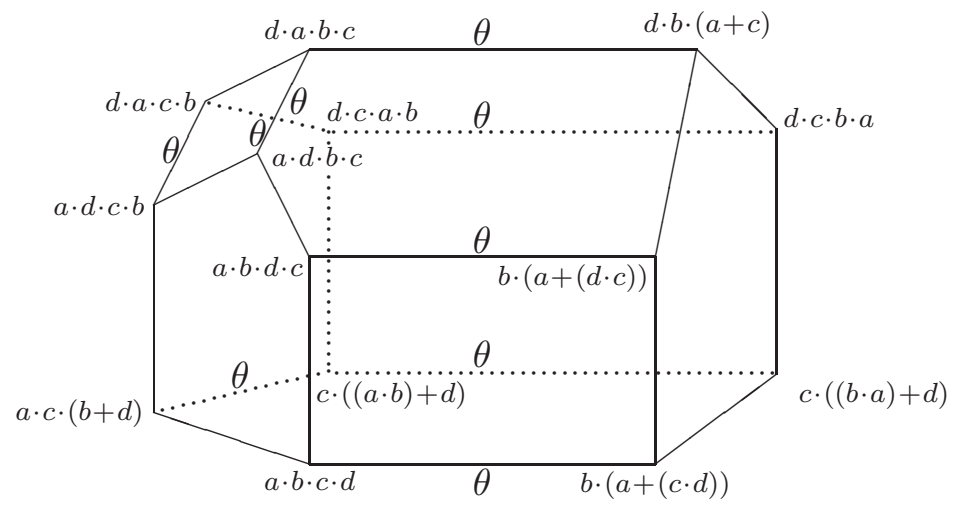


whose edges with $\theta$ stand for $\theta$ arrows, while the remaining edges stand for $\beta$ arrows.

Example 13.6. Let $G$ and $\alpha_{G}$ be as in Examples 13.1 13.3 and the preceding example. Let $X=\{111,112,12,21,221,222\}$. Let $a, b, c$ and $d$ stand respectively for $11,1,2$ and 22 , which are the inner vertices of the following binary tree, whose leaves make $X$ :
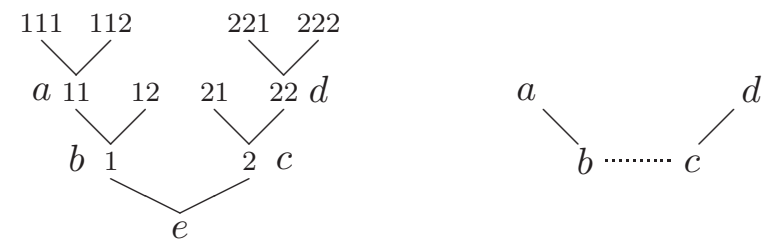

On the right of our tree one finds the graph of the inner vertices.

The objects of $\mathcal{W O}_{e}^{-}(X)$ correspond bijectively to the vertices of the following threedimensional associahedron:

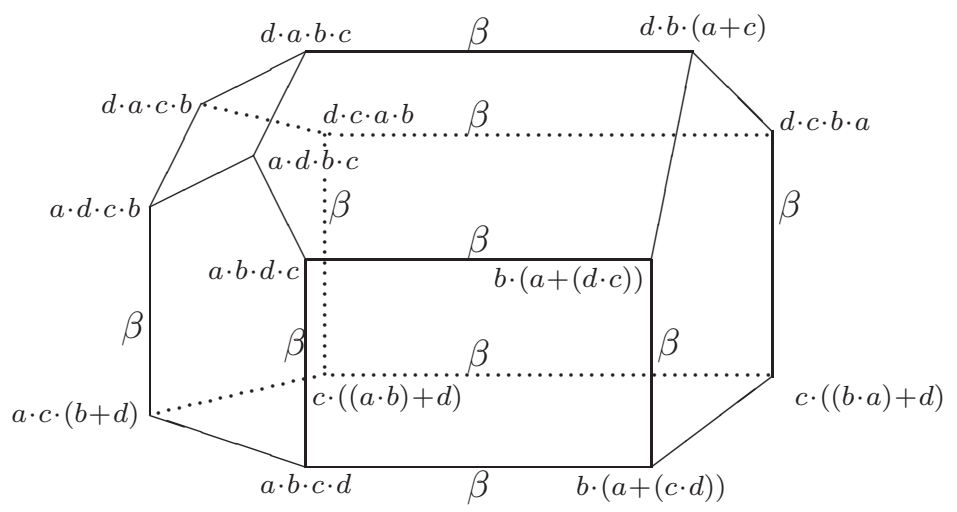

whose edges with $\beta$ stand for $\beta$ arrows, while the remaining edges stand for $\theta$ arrows.

Example 13.7. Let $G=\{x, y\}$, and let $\alpha_{G}(x)=2$ and $\alpha_{G}(y)=3$. Let $X=\{111,112,121$, $122,131,132,2\}$. Let $a, b, c$ and $d$ stand respectively for $1,11,12$ and 13 , which are the inner vertices of the following tree, whose leaves make $X$ :
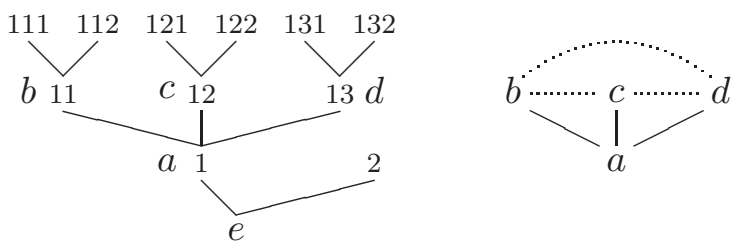

On the right of our tree one finds the graph of the inner vertices.

The objects of $\mathcal{W O}_{e}^{-}(X)$ correspond bijectively to the vertices of the following threedimensional permutohedron: 


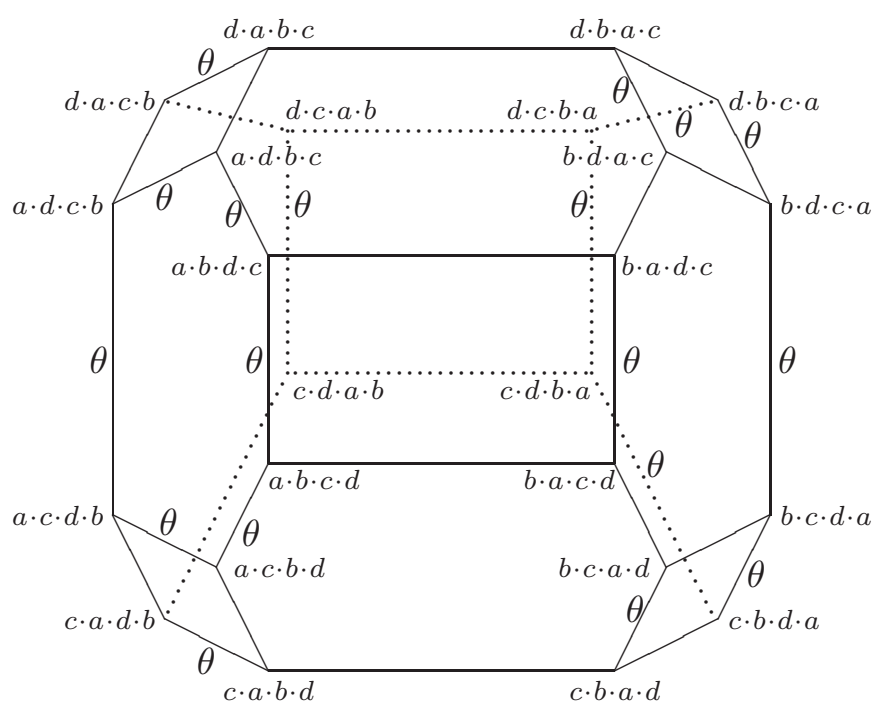

whose edges with $\theta$ stand for $\theta$ arrows, while the remaining edges stand for $\beta$ arrows.

Example 13.8. Let $G$ and $\alpha_{G}$ be as in Examples 13.1]13.3 and 13.5]13.6, Let $X=$ $\{11111,11112,1112,112,12,2\}$. Let $a, b, c$ and $d$ stand respectively for $1,11,111$ and 1111 , which are the inner vertices of the following binary tree, whose leaves make $X$ :
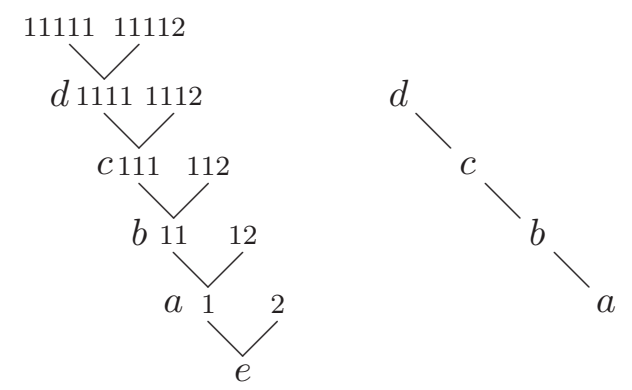

On the right of our tree one finds the graph of the inner vertices.

The objects of $\mathcal{W O}_{e}^{-}(X)$ correspond bijectively to the vertices of the three-dimensional associahedron whose edges all stand for $\beta$ arrows (its picture is like that for Examples 13.5 and 13.6 without the labels $\theta$ and $\beta$ ).

Example 13.9. Let $G=\{x, y\}$, and let $\alpha_{G}(x)=2$ and $\alpha_{G}(y)=4$. Let $X=\{11,12,21,22$, $31,32,41,42\}$. Let $a, b, c$ and $d$ stand respectively for $1,2,3$ and 4 , which are the inner vertices of the following tree, whose leaves make $X$ :
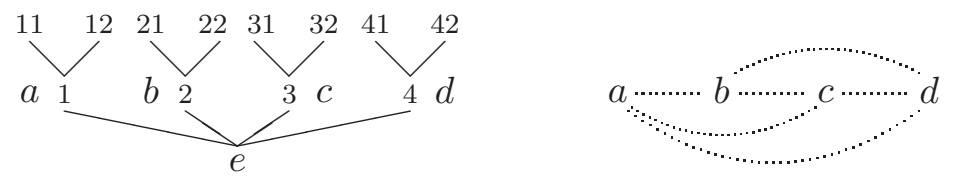

On the right of our tree one finds the graph of the inner vertices.

The objects of $\mathcal{W O}_{e}^{-}(X)$ correspond bijectively to the vertices of the three-dimensional permutohedron whose edges all stand for $\theta$ arrows (its picture is like that for Example 13.7 without labels for edges). 


\section{PART III}

\section{Coherence of $M_{0} n_{u}$}

In [MLP85] (Section 2) it was established that the notion of bicategory is coherent, in the sense that all diagrams of canonical arrows commute. The proof of this coherence result is obtained by imitating the proof of monoidal coherence (see MLa63, MLa98, Section VII.2).

Our purpose in this, concluding part, of the paper is to prove an analogous coherence result for our notion of weak Cat-operad, which is analogous to the notion of bicategory. This amounts to showing that the category $\mathcal{W O}_{e}$ is a preorder (i.e. that all diagrams commute in this category, or that for a given source and target there is not more than one arrow). This coherence result for $\mathcal{W} \mathcal{O}_{e}$ does not rely only on monoidal coherence, as coherence for bicategories does. Besides relying on monoidal coherence, it relies also on a generalization of coherence for symmetric monoidal categories, which is related to the presentation of structures related to symmetric groups.

Coherence for $\mathcal{W O}_{e}$ is a justification of our definition of this category, and of our notion of weak Cat-operad. We will establish the coherence of $\mathcal{W O}_{e}$ by establishing the coherence of $\mathcal{W} \mathcal{O}_{u}$. We first introduce a category $M_{o n}$ derived from $\mathcal{W} \mathcal{O}_{u}$, which is analogous to a monoidal category.

The category $\mathrm{Mon}_{u}$ is defined like $\mathcal{W} \mathcal{O}_{u}$ save that we omit the basic arrow terms $\theta_{h, g, f}$ and the axiomatic equations that involve $\theta$ explicitly (these are $(\theta$ nat $),(\theta \theta),(\theta$ YB $),(\beta \theta 1)$ and $(\beta \theta 2))$. The remaining axiomatic equations are analogous to Mac Lane's postulates for monoidal categories (see [MLa63] and [MLa98], Section VII.1); the difference is that $\triangleleft$ is a partial operation on objects and on arrows, and is hence not a real biendofunctor, though it is analogous to such a functor.

Let an arrow term of $\mathrm{Mon}_{u}$ be called directed when $\beta, \mu^{-1}$ and $\lambda^{-1}$ do not occur in it (but $\beta^{-1}, \mu$ and $\lambda$ may occur). Let us call an object $f$ of $M o n_{u}$, i.e. term of $\mathcal{O}_{u}$, normal when either all parentheses in $f$ are associated to the left and $\mathbf{I}$ does not occur in $f$, or $f$ is of the form $a \cdot \mathbf{I}$. One can then prove the following.

Lemma 14.1. If $u$ and $v$ are directed arrow terms of $M_{0} n_{u}$ of the same type with a normal target, then $u=v$ in $M_{\text {on }}$.

Proof. The proof of this lemma is obtained by imitating a part of the proof of monoidal coherence in MLa98 (Section VII.2, Theorem 1; see also DP04, Sections 4.2, 4.3 and 4.6, Directedness Lemmata). There is nothing essentially new in this inductive proof, which consists in showing a kind of confluence property, related to what one has in term-rewriting. For example, if $u$ and $v$ are respectively of the form $u^{\prime} \circ\left(\mathbf{1}_{j} \triangleleft \beta_{h, g, f}^{-1}\right)$ and $v^{\prime} \circ \beta_{j, h, g \triangleleft f}^{-1}$, then, since the target of $u$ and $v$ is normal, there is a $w$ such that by the induction hypothesis

$$
\begin{aligned}
& u^{\prime}=w \circ\left(\beta_{j, h, g}^{-1} \triangleleft \mathbf{1}_{f}\right) \circ \beta_{j, h \triangleleft g, f}^{-1}, \\
& v^{\prime}=w \circ \beta_{j, h, g \triangleleft f}^{-1} ;
\end{aligned}
$$

and then by using the equation obtained from $(\beta$ pent $)$ and $(\beta \beta)$, analogous to Mac Lane's pentagonal diagram, we obtain $u=v$. 
We can then establish the following.

Proposition 14.2. $\mathrm{Mon}_{u}$ is a preorder.

To prove this proposition we may proceed as for Associative Coherence in [DP04] (Section 4.3).

\section{The CATEgory $\mathcal{W} \mathcal{O}_{u}^{\theta}$}

Next we define a category $\mathcal{W O}_{u}^{\theta}$, which is $\mathcal{W O}_{u}$ strictified in the monoidal structure. This means that in $\mathcal{W} \mathcal{O}_{u}^{\theta}$ the arrows $\beta, \mu$ and $\lambda$, and their inverses, become all identity arrows, and the monoidal structure of $\mathcal{W O}_{u}^{\theta}$ is trivial. As in the case of monoidal categories, and as in [DP04] (Section 3.2), by relying on Proposition 14.2 one can show that $\mathcal{W} \mathcal{O}_{u}$ and $\mathcal{W} \mathcal{O}_{u}^{\theta}$ are equivalent categories.

Now we will define $\mathcal{W} \mathcal{O}_{u}^{\theta}$ syntactically. Its objects are the normal terms of $\mathcal{O}_{u}$ as defined in the preceding section. We may identify these terms by terms where $\triangleleft$ and parentheses are deleted, and in which $\mathbf{I}$ does not occur, except if the term is of the form $a \cdot \mathbf{I}$. We use this abbreviated notation below.

As basic arrow terms we have the following:

$$
\begin{aligned}
& \mathbf{1}_{f}: f \rightarrow f, \quad \text { for every object } f, \\
& \theta_{h, g, f}: h g f \rightarrow h f g, \quad \text { provided } t(f) \in s(h) \text { and } t(g) \in s(h) .
\end{aligned}
$$

Next we have the operations on arrow terms as for $\mathcal{W} \mathcal{O}_{u}$ (see Section 7) save that $v \triangleleft u$ : $g \triangleleft f \rightarrow g^{\prime} \triangleleft f^{\prime}$ is written $v u: g f \rightarrow g^{\prime} f^{\prime}$. (We write $v \circ u$ as before.)

Besides $u=u$ and the categorial equations, and the equations (ins 1), (ins 2), ( $\theta$ nat), $(\theta \theta)$ and $(\theta \mathrm{YB})$ (all written without $\triangleleft)$, the axiomatic equations of $\mathcal{W} \mathcal{O}_{u}^{\theta}$ are the following strictified versions of $(\beta \theta 1)$ and $(\beta \theta 2)$ :

$$
\begin{aligned}
& \mathbf{1}_{j} \theta_{h, g, f}=\theta_{j h, g, f}, \\
& \theta_{j, h g, f}=\left(\theta_{j, h, f} \mathbf{1}_{g}\right) \circ \theta_{j h, g, f} .
\end{aligned}
$$

(Note that if the left-hand sides of these equations are legitimate, then the right-hand sides are legitimate too, but not conversely.)

The rules of the equational axiomatic system are the same as for $\mathcal{W} \mathcal{O}_{u}$. This concludes the definition of the category $\mathcal{W} \mathcal{O}_{u}^{\theta}$.

\section{6. $\mathcal{C}_{\Gamma} \operatorname{AND} \mathcal{B C}_{\Gamma}$}

We introduce a family of categories we call $\mathcal{C}_{\Gamma}$ and show in the next section that every category in the family is coherent in a sense to be made precise. A particular category in the family, for a particular choice of $\Gamma$, will be shown isomorphic to the category $\mathcal{W O}_{u}^{\theta}$ of the preceding section, and this will establish that $\mathcal{W} \mathcal{O}_{u}^{\theta}$ is coherent, which in this particular case implies that $\mathcal{W O}_{u}^{\theta}$ is a preorder. Symmetric groups arise as particular members of this family, and the proof that $\mathcal{C}_{\Gamma}$ is coherent will proceed as a proof that would show the completeness of a standard presentation of symmetric groups (see the next section).

Now we introduce $\mathcal{C}_{\Gamma}$. Let $A, B, P, Q, R, S, U, \ldots$, perhaps with indices, stand for finite (possibly empty) sequences, i.e. for words, in an alphabet whose members we call atoms; we use $p, q, r, \ldots$, perhaps with indices, for atoms. We use $e$, as before, for the empty word. The set of objects of $\mathcal{C}_{\Gamma}$ is some set of these words, not necessarily all. (So the objects of $\mathcal{C}_{\Gamma}$ make a language.) 
The basic arrow terms of $\mathcal{C}_{\Gamma}$ make a set $\Gamma$, which satisfies the following. For every object $A$, the arrow term $A: A \rightarrow A$ is in $\Gamma$ (we abbreviate $\mathbf{1}_{A}$ by writing just $A$ ). We have in $\Gamma$ also some arrow terms of the form

$$
A[p, q] B: A p q B \rightarrow A q p B
$$

provided both the source and the target are objects. All the arrow terms in $\Gamma$ are of these two kinds. Finally, $\Gamma$ must satisfy the following condition:

$(\Gamma)$ if the basic arrow terms on one side of the equations $(C 1)$ and $(C 2)$ below are in $\Gamma$, then all the basic arrow terms on the other side of these equations are in $\Gamma$ too.

(It is natural to call the arrow terms $A: A \rightarrow A$ basic, though they could have been left out from $\Gamma$, and introduced a bit later, to produce $\mathcal{C}_{\Gamma}$.)

All the arrow terms of $\mathcal{C}_{\Gamma}$ are defined by starting from $\Gamma$, and closing under composition: if $u: A \rightarrow B$ and $v: B \rightarrow C$ are arrow terms, then $v \circ u: A \rightarrow C$ is an arrow term. We use $u$, $v, w, \ldots$, perhaps with indices, for arrow terms, and we use the abbreviation given by the following inductive clause:

$$
A(v \circ u) B={ }_{d f} A v B \circ A u B .
$$

Besides $u=u$ and the categorial equations $u \circ A=u=B \circ u$, for $u: A \rightarrow B$, and $(w \circ v) \circ u=w \circ(v \circ u)$, the axiomatic equations of $\mathcal{C}_{\Gamma}$ are the following:

$$
\begin{aligned}
& A(s r U[p, q] \circ[r, s] U p q) B=A([r, s] U q p \circ r s U[p, q]) B, \\
& A(s[p, r] \circ[p, s] r \circ p[r, s]) B=A([r, s] p \circ r[p, s] \circ[p, r] s) B, \\
& A([p, r] \circ[r, p]) B=A r p B .
\end{aligned}
$$

As rules we have symmetry and transitivity of $=$ and congruence for $\circ$ (see Section 7 ). This concludes our definition of the equations of $\mathcal{C}_{\Gamma}$, and of the category $\mathcal{C}_{\Gamma}$.

The axiomatic equations of $\mathcal{C}_{\Gamma}$ are analogous to the equations of the standard presentation of the symmetric group $S_{n}$, for $n \geq 1$, with the generators $\sigma_{i}$, for $1 \leq i<n$, being the transpositions of $i$ and $i+1$ (see CM57, Section 6.2). The equation $(C 1)$ corresponds to the permutability of $\sigma_{i}$ and $\sigma_{j}$ when $i-j$ is at least 2. The equation $(C 2)$ corresponds to the equation

$$
\sigma_{i+1} \circ \sigma_{i} \circ \sigma_{i+1}=\sigma_{i} \circ \sigma_{i+1} \circ \sigma_{i}
$$

(YB comes from Yang-Baxter), and (C3) corresponds to the $\sigma_{i}$ 's being self-inverse.

The symmetric group $S_{n}$ is $\mathcal{C}_{\Gamma}$ that has a unique object $p^{n}$, which is a sequence of $n$ occurrences of $p$, and the set $\Gamma$ is made of the arrow terms $p^{i-1}[p, p] p^{k}$, where $i \geq 1, k \geq 0$ and $i+1+k=n$, which correspond to $\sigma_{i}$. Note that in general $\mathcal{C}_{\Gamma}$ is not a group. It need not even be a groupoid (in the categorial sense, a Brandt groupoid; see [MLa98, Section I.5); we may have $A[p, q] B$ in $\Gamma$ without having its inverse $A[q, p] B$.

To reduce the arrow terms of $\mathcal{C}_{\Gamma}$ to normal form we introduce the category $\mathcal{B C}_{\Gamma}$, a variant of $\mathcal{C}_{\Gamma}$, which we will show isomorphic to $\mathcal{C}_{\Gamma}$. The objects of $\mathcal{B C}_{\Gamma}$ are those of $\mathcal{C}_{\Gamma}$. The arrow terms of $\mathcal{B C}_{\Gamma}$ are defined starting from the same basic arrow terms $\Gamma$, and closing under composition and under the following:

$(\dagger) \quad$ if for every $s$ in the word $S$, such that $S$ is $S^{\prime} s S^{\prime \prime}$, the arrow term

$$
A S^{\prime}[r, s] S^{\prime \prime} B: A S^{\prime} r s S^{\prime \prime} B \rightarrow A S^{\prime} s r S^{\prime \prime} B
$$

is in $\Gamma$, then

$$
A[r, S] B: A r S B \rightarrow A S r B
$$

is an arrow term. 
Note that according to this clause $A[r, e] B: A r B \rightarrow A r B$ is always an arrow term. Note also that according to this definition we also have the implication converse to $(\dagger)$.

The equations of $\mathcal{B C}_{\Gamma}$ are defined like those of $\mathcal{C}_{\Gamma}$ save that the axiomatic equations $(C 1),(C 2)$ and $(C 3)$ are replaced by the following axiomatic equations:

$(B C 1) \quad A(S r U[p, Q] \circ[r, S] U p Q) B=A([r, S] U Q p \circ r S U[p, Q]) B$, provided neither $Q$ nor $S$ is $e$,

$(B C 2) \quad A([p, Q S r U] \circ p Q[r, S] U) B=A(Q[r, S] U p \circ[p, Q r S U]) B, \quad$ provided $S$ is not $e$,

$(B C 3) \quad A(S[p, U r Q] \circ[r, S p U] Q) B=A([r, S U] Q p \circ r S[p, U Q]) B$,

(BC4) $A(S[r, Q] \circ[r, S] Q) B=A[r, S Q] B$, provided neither $Q$ nor $S$ is $e$,

(BC5) $A[r, e] B=A r B$.

This concludes the definition of $\mathcal{B C}_{\Gamma}$.

The axiomatic equations of $\mathcal{B C}_{\Gamma}$ are analogous to the equations that may be found in DP04 (Section 5.2). These equations are such as to enable us to reach quickly a normal form for arrow terms, with which we will deal in the next section. In the remainder of this section we will establish that $\mathcal{C}_{\Gamma}$ and $\mathcal{B C}_{\Gamma}$ are isomorphic.

We show first that we have in $\mathcal{C}_{\Gamma}$ the structure of $\mathcal{B C}_{\Gamma}$. We define $A[r, S] B$ in $\mathcal{C}_{\Gamma}$ by the following inductive clauses:

$$
\begin{aligned}
& A[r, e] B=_{d f} A r B, \\
& A[r, s Q] B=_{d f} A(s[r, Q] \circ[r, s] Q) B .
\end{aligned}
$$

Then it remains to derive the equations $(B C 1)-(B C 5)$ in $\mathcal{C}_{\Gamma}$.

We derive first $(B C 1)$ by induction on the sum $n$ of the lengths of $Q$ and $S$. In the basis when $n$ is 2 , we use $(C 1)$, and in the induction step we just use the induction hypothesis.

Next we derive by induction on the length $n$ of $S$ the equation $(C 2 S)$, which is $(C 2)$ with $s$ replaced by $S$. The basis, when $n$ is 0 , is trivial. In the induction step, to derive our equation (read from left to right) we use ( $B C 1)$ (read from left to right), the induction hypothesis and $(C 2)$ (read from left to right).

To derive $(B C 2)$ (read from left to right) we use $(B C 1)$ (read in both directions) and $(C 2 S)$ (read from left to right).

To derive $(B C 3)$ (read from left to right) we use $(C 2 S),(C 3)$ and $(B C 1)$ (all read from left to right).

The equations $(B C 4)$ and $(B C 5)$ hold in $\mathcal{C}_{\Gamma}$ by definition. This establishes that we have the structure of $\mathcal{B C}_{\Gamma}$ in $\mathcal{C}_{\Gamma}$.

We have noted above in parentheses when we needed the equations $(C 2)$ and $(C 3)$ only from left to right (while $(C 1)$, via $(B C 1)$, is needed in both directions). This may be interesting when our procedure is connected with the reduction procedure of [Laf03] (Section 2.1 and Appendix A).

To establish the converse - namely, that we have the structure of $\mathcal{C}_{\Gamma}$ in $\mathcal{B C}_{\Gamma}$ - is an easy matter. The equations $(C 1),(C 2)$ and $(C 3)$ amount to particular cases of $(B C 1)$, $(B C 2)$ and $(B C 3)$, with the help of $(B C 4)$ and $(B C 5)$. The definitions of $A[r, e] B$ and $A[r, s Q] B$, which we introduced in $\mathcal{C}_{\Gamma}$, clearly hold in $\mathcal{B C}_{\Gamma}$ by $(B C 5)$ and $(B C 4)$. So we may conclude that $\mathcal{C}_{\Gamma}$ and $\mathcal{B C}_{\Gamma}$ are isomorphic.

As a consequence of the isomorphism of $\mathcal{C}_{\Gamma}$ and $\mathcal{B C}_{\Gamma}$ we obtain that if on one side of the equations $(B C 1)-(B C 5)$ we have arrow terms of $\mathcal{B C}_{\Gamma}$, then on the other side we have such arrow terms too. The straightforward proof of that is based essentially on $(\dagger)$, the 
implication converse to $(\dagger)$, and the fact that if on one side of an equation of $\mathcal{C}_{\Gamma}$ we have arrow terms of $\mathcal{C}_{\Gamma}$, then on the other side we have such arrow terms too.

\section{Coherence of $\mathcal{C}_{\Gamma}$}

We say that an arrow term of $\mathcal{B C}_{\Gamma}$ is in normal form when it is of the form

$$
A_{1} Q_{1} p_{1} B_{1} \circ A_{1}\left[p_{1}, Q_{1}\right] B_{1} \circ \ldots \circ A_{n}\left[p_{n}, Q_{n}\right] B_{n},
$$

for $n \geq 0$, for the words $Q_{1}, \ldots, Q_{n}$ nonempty and for

$$
\left|B_{1}\right|>\left|B_{2}\right|>\ldots>\left|B_{n}\right| \text {, }
$$

where $\left|B_{i}\right|$ is the length of $B_{i}$. This, or an analogous normal form, for symmetric groups is implicit in Mo896] and Bur11 (Note C), and occurs explicitly in Laf95] (Section 3.2), Laf03. (Section 2.1) and DP04] (Section 5.2).

A survey of all possible cases shows that if an arrow term of $\mathcal{B C}_{\Gamma}$ is not in normal form, then it has, after perhaps applying categorial equations, a subterm of the form of the left-hand side of one of the equations $(B C 1)-(B C 5)$, and hence one of these equations may be applied. We can then establish the following.

Lemma 17.1. Every arrow term of $\mathcal{B C}_{\Gamma}$ is equal in $\mathcal{B C}_{\Gamma}$ to an arrow term in normal form.

Proof. For every arrow term of the form

$$
C_{m}\left[r_{m}, S_{m}\right] D_{m} \circ \ldots \circ C_{1}\left[r_{1}, S_{1}\right] D_{1},
$$

where $m \geq 2$, consider the following measure of this arrow term:

$$
\sum_{i=1}^{m}\left(\left|C_{i}\right|+1+\left|S_{i}\right|\right) \cdot i .
$$

Then it can be checked that with each application of $(B C 1)-(B C 4)$ from left to right the measure decreases. The equation $(B C 5)$ from left to right works together with the categorial equations $u \circ A=u=B \circ u$ to reduce our measure.

The graph of an arrow term of $\mathcal{B C}_{\Gamma}$ is derived from a bijection between finite ordinals, defined as for symmetric groups or as for symmetric monoidal categories (see [DP04]). This bijection induces a graph with edges connecting an occurrence of an atom in the source to an occurrence of the same atom in the target. The graphs of $A[p, q] B, A[p, Q] B$ and of the identity arrow $A: A \rightarrow A$ are given by
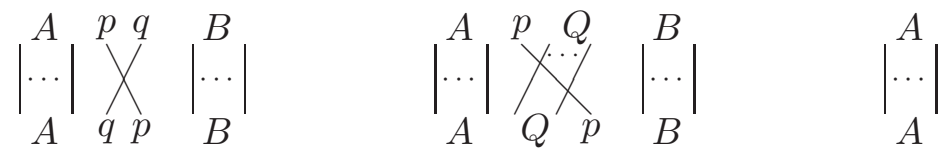

The graphs for arrow terms with $\circ$ are obtained by composing the underlying bijections. We can then prove the following.

Lemma 17.2. If the arrow terms $u, v: A \rightarrow B$ of $\mathcal{B C}_{\Gamma}$ are in normal form and their graphs are the same, then $u$ and $v$ are the same arrow term.

Proof. The proof of this lemma is analogous to the proof of the Uniqueness Lemma in DP04 (Section 5.2), to which we refer for details. Here is a sketch of the proof.

Let $u$ and $v$ be respectively the arrow terms 


$$
\begin{aligned}
& A \circ A_{1}\left[p_{1}, Q_{1}\right] B_{1} \circ \ldots \circ A_{n}\left[p_{n}, Q_{n}\right] B_{n}, \\
& A \circ C_{1}\left[r_{1}, S_{1}\right] D_{1} \circ \ldots \circ C_{m}\left[r_{m}, S_{m}\right] D_{m}
\end{aligned}
$$

in normal form. We proceed by induction on $n$. If $n=0$, then we show that $m$ must be 0 too; otherwise the graphs would differ.

If $n>0$, then we must have $m>0$ too, as we have just shown, and $A_{n}\left[p_{n}, Q_{n}\right] B_{n}$ must be equal to $C_{m}\left[r_{m}, S_{m}\right] D_{m}$; otherwise the graphs would differ in the edges of $p_{n}$ and $r_{m}$ :
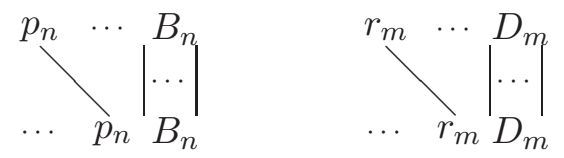

(see [DP04], Section 5.2, for details). We then conclude that

$$
\begin{aligned}
& A \circ A_{1}\left[p_{1}, Q_{1}\right] B_{1} \circ \ldots \circ A_{n-1}\left[p_{n-1}, Q_{n-1}\right] B_{n-1}, \\
& A \circ C_{1}\left[r_{1}, S_{1}\right] D_{1} \circ \ldots \circ C_{m-1}\left[r_{m-1}, S_{m-1}\right] D_{m-1}
\end{aligned}
$$

must have the same graph, and we apply the induction hypothesis to them.

Then we obtain the following

Proposition 17.3. For $u$ and $v$ arrow terms of $\mathcal{B C}_{\Gamma}$ of the same type we have $u=v$ in $\mathcal{B C}_{\Gamma}$ iff $u$ and $v$ have the same graph.

Proof. It is easy to establish the implication from left to right by induction on the length of derivation. For the converse implication, we have by Lemma 17.1 that $u=u^{\prime}$ and $v=v^{\prime}$ in $\mathcal{B C}_{\Gamma}$ for $u^{\prime}$ and $v^{\prime}$ in normal form. From the assumption that $u$ and $v$ have the same graph we conclude, by the implication from left to right, that $u^{\prime}$ and $v^{\prime}$ have the same graph. But then by Lemma 17.2 we have that $u^{\prime}$ is $v^{\prime}$, and hence $u=v$ in $\mathcal{B C}_{\Gamma}$ by symmetry and transitivity of $=$.

From Proposition 17.3 from left to right and Lemma 17.2 we may infer that for every arrow term $u$ of $\mathcal{B C}_{\Gamma}$ there is a unique arrow term $u^{\prime}$ of $\mathcal{B C}_{\Gamma}$ in normal form such that $u=u^{\prime}$ in $\mathcal{B C}_{\Gamma}$. Note that we did not need this kind of uniqueness proposition to establish coherence. Note also that we did not establish this uniqueness proposition by means like confluence of term rewriting. Instead we have a uniqueness proposition, our Lemma 17.2, which involves graphs. Establishing uniqueness in the latter way may often be more easier than doing it in the former one. The paper Laf03. (Section 2.1 and Appendix A) considers uniqueness of normal form established via confluence of term rewriting.

As an alternative to the style of proof of coherence of this paper there is the style of the original paper of [Mo896], which one finds also in Bur11. This style works for symmetric groups, and relies on the fact that in that case one can establish that for the symmetric group $S_{n}$ there are $n$ ! normal forms and $n$ ! permutations. Then it is enough to establish that the map from the syntax for $S_{n}$ to permutations is onto, which means that every permutation is represented by a term of $S_{n}$, i.e. a composition of generators. (We must also establish that the map in question is a homomorphism, which means that every equation of the syntax holds for permutations.) It follows then that the map is one-one.

This old style of argument seems however too complicated and chaotic in the case of $\mathcal{C}_{\Gamma}$. It may work for a regularly chosen $\Gamma$, but $\Gamma$ may be irregular, and it is not clear with what the number $n$ ! can be replaced. It would be a number lesser than or equal to $n$ !, but it may change irregularly, and preclude an inductive argument on $n$. 
Since $\mathcal{C}_{\Gamma}$ and $\mathcal{B C}_{\Gamma}$ are isomorphic, we may establish Proposition 17.3 for $\mathcal{C}_{\Gamma}$, and we call that proposition the Coherence of $\mathcal{C}_{\Gamma}$. (The graphs for $\mathcal{C}_{\Gamma}$ are obtained in an obvious manner through the isomorphism with $\mathcal{B C}_{\Gamma}$.)

\section{8. $\mathcal{C}_{\Gamma}$ AND $\mathcal{W} \mathcal{O}_{u}^{\theta}-$ COHERENCE OF $\mathcal{W} \mathcal{O}_{u}^{\theta}$}

Let us now show that $\mathcal{W O}_{u}^{\theta}$ may be conceived as a category $\mathcal{C}_{\Gamma}$. The atoms are all the terms $a \cdot x$ and $a \cdot \mathbf{I}$ of $\mathcal{O}_{u}$. The objects of $\mathcal{W} \mathcal{O}_{u}^{\theta}$ are the normal terms of $\mathcal{O}_{u}$, which may be identified with some words made of atoms. The set $\Gamma$ is made first of the arrow terms $\mathbf{1}_{f}$ for every object $f$ of $\mathcal{W} \mathcal{O}_{u}^{\theta}$; here $\mathbf{1}_{f}$ stands for $f: f \rightarrow f$. Next we have in $\Gamma$ all the arrow terms $\theta_{h, a \cdot x, b \cdot y} \mathbf{1}_{j}$ and $\theta_{h, a \cdot x, b \cdot y}$ of $\mathcal{W} \mathcal{O}_{u}^{\theta}$; they stand for

$$
h[a \cdot x, b \cdot y] j: h(a \cdot x)(b \cdot y) j \rightarrow h(b \cdot y)(a \cdot x) j
$$

and the same without $j$.

We use (ins 1), (ins 2), ( $\theta 1)$ and $(\theta 2)$ to reduce every arrow term $u$ of $\mathcal{W} \mathcal{O}_{u}^{\theta}$ to the form $u_{n} \circ \ldots \circ u_{1}$ where each $u_{i}$ is either an identity $f: f \rightarrow f$, or $\theta_{h, a \cdot x, b \cdot y} \mathbf{1}_{j}$, or $\theta_{h, a \cdot x, b \cdot y}$. We check next that we have the equations of $\mathcal{C}_{\Gamma}$ in $\mathcal{W} \mathcal{O}_{u}^{\theta}$. We have of course $u=u$ and the categorial equations. For $(C 1)$ we use essentially $(\theta$ nat), for $(C 2)$ we use essentially $(\theta \mathrm{YB})$, and for $(C 3)$ we use essentially $(\theta \theta)$; for all that we need also (ins 2$)$. To show that, conversely, all the equations of $\mathcal{W} \mathcal{O}_{u}^{\theta}$ may be derived from the $\mathcal{C}_{\Gamma}$ assumptions is a consequence of the coherence of $\mathcal{C}_{\Gamma}$. Finally, we check easily that the set $\Gamma$ of $\mathcal{W} \mathcal{O}_{u}^{\theta}$ satisfies condition $(\Gamma)$ (see Section 16).

So $\mathcal{W} \mathcal{O}_{u}^{\theta}$ is a $\mathcal{C}_{\Gamma}$ category, and hence coherence for $\mathcal{C}_{\Gamma}$ holds for it. But in this particular case coherence for $\mathcal{C}_{\Gamma}$ becomes the following.

Proposition 18.1. For every arrow terms $u$ and $v$ of $\mathcal{W} \mathcal{O}_{u}^{\theta}$ of the same type we have $u=v$ in $\mathcal{W} \mathcal{O}_{u}^{\theta}$.

In other words, the category $\mathcal{W O}_{u}^{\theta}$ is a preorder. This proposition is a consequence of the coherence of $\mathcal{C}_{\Gamma}$ and of the fact that in $\mathcal{W} \mathcal{O}_{u}^{\theta}$ the type of an arrow term determines uniquely the graph. The reason for that is that in the set $\Gamma$ of $\mathcal{W} \mathcal{O}_{u}^{\theta}$ we do not have $h[a \cdot x, a \cdot x] j$ and $h[a \cdot x, a \cdot x]$.

From Proposition 18.1 and the equivalence of $\mathcal{W} \mathcal{O}_{u}$ with $\mathcal{W} \mathcal{O}_{u}^{\theta}$ we may conclude that $\mathcal{W O} \mathcal{O}_{u}$ is a preorder. From that and from Proposition [11.6 we conclude that the category $\mathcal{W O}_{e}$ is a preorder. This establishes the coherence of our notion of weak Cat-operad of Section 12. This notion is coherent in the same sense in which Mac Lane' s notion of monoidal category and the notion of bicategory are coherent. All diagrams of canonical arrows commute in it.

\section{ACKNowledgment}

Work on this paper was supported by the Ministry of Science of Serbia (Grant ON174026). 


\section{REFERENCES}

[Bén63] J. Bénabou. Catégories avec multiplication. C. R. Acad. Sci., Paris, Sr. I, Math., 256:1887-1890, 1963.

[Bur11] W. Burnside. Theory of Groups of Finite Order, second edition, Cambridge University Press, Cambridge, 1911 (reprint, Dover, New York, 1955).

[CK73] C.C. Chang and H.J. Keisler. Model Theory, North-Holland, Amsterdam, 1973.

[CM57] H.S.M. Coxeter and W.O.J. Moser. Generators and Relations for Discrete Groups, Springer, Berlin, 1957.

[DS01] B. Day and R. Street. Lax monoids, pseudo-operads and convolution. Diagrammatic Morphisms and Applications (D.E. Radford et al., editors), Contemp. Math., 318:75-96, 2003.

[DP04] K. Došen and Z. Petrić. Proof-Theoretical Coherence, KCL Publications (College Publications), London, 2004 (revised version of 2007 available at: http://www.mi.sanu.ac.rs/ kosta/coh.pdf).

[DP06] K. Došen and Z. Petrić. Associativity as commutativity. J. Symb. Log., 71:217-226, 2006 (available at: $a r X i v)$.

[DP06a] K. Došen and Z. Petrić. Medial commutativity. Ann. Pure Appl. Logic, 146:237-255, 2007 (available at: $\operatorname{arXiv).}$

[DP07] K. Došen and Z. Petrić. Coherence and confluence. Perspectives on Universal Logic (J.-Y. Béziau and A. Costa-Leite, editors), Polimetrica, Monza, 205-215, 2007 (available at: arXiv).

[DP11] K. Došen and Z. Petrić. Hypergraph polytopes. Topology Appl., 158:1405-1444, 2011 (available at: arXiv).

[DP10] K. Došen and Z. Petrić. Shuffles and concatenations in constructing of graphs. Math. Struct. Comput. Sci., 22:904-930, 2012 (available at: arXiv).

[DP12] K. Došen and Z. Petrić. Intermutation. Appl. Categ. Struct., 20:43-95, 2012 (available at: arXiv).

[Gen35] G. Gentzen. Untersuchungen über das logische Schließen. Math. Z., 39:176-210, 405-431, 1935 (English translation: Investigations into logical deduction, in The Collected Papers of Gerhard Gentzen, M.E. Szabo, editor, North-Holland, Amsterdam, 68-131, 1969).

[GR63] G.Th. Guilbaud and P. Rosenstiehl. Analyse algébrique d'un scrutin. Math. Sci. Hum., 4:9-33, 1963.

[Har69] F. Harary. Graph Theory, Addison-Wesley, Reading, Mass., 1969.

[Kap93] M.M. Kapranov. The permutoassociahedron, Mac Lane's coherence theorem and asymptotic zones for the KZ equation. J. Pure Appl. Algebra, 85:119-142, 1993.

[Laf95] Y. Lafont. Equational reasoning with 2-dimensional diagrams. Term Rewriting (H. Comon and J.-P. Jouannaud, editors), Lect. Notes Comput. Sci., 909:170-195, 1995.

[Laf03] Y. Lafont. Towards an algebraic theory of Boolean circuits. J. Pure Appl. Algebra, 184:257-310, 2003.

[Lam69] J. Lambek. Deductive systems and categories II: Standard constructions and closed categories. Category Theory, Homology Theory and their Applications I, Lect. Notes Math., 86:76-122, 1969.

[Lam89] J. Lambek. Multicategories revisited. Categories in Computer Science and Logic (J.W. Gray and A. Scedrov, editors), American Mathematical Society, Providence, 217-239, 1989.

[Lei04] T. Leinster. Higher Operads, Higher Categories, Cambridge University Press, Cambridge, 2004.

[Lod97] J.-L. Loday et al., editors, Operads: Proceedings of Renaissance Conferences, Contemp. Math., 202, 1997.

[MLa63] S. Mac Lane. Natural associativity and commutativity. Rice Univ. Stud., 49:28-46, 1963.

[MLa98] S. Mac Lane. Categories for the Working Mathematician, expanded second edition, Springer, Berlin, 1998.

[MLP85] S. Mac Lane and R. Paré. Coherence for bicategories and indexed categories. J. Pure Appl. Algebra, 37:59-80, 1985.

[MSS02] M. Markl, S. Shnider and J.D. Stasheff. Operads in Algebra, Topology and Physics, American Mathematical Society, Providence, 2002.

[May72] J.P. May. The Geometry of Iterated Loop Spaces, Lect. Notes Math., 271, 1972.

[Mo896] E.H. Moore. Concerning the abstract groups of order $k$ ! and $\frac{1}{2} k$ ! holohedrically isomorphic with the symmetric and alternating substitution-groups on $k$ letters. Proc. Lond. Math. Soc., 28:357-366, 1896.

[Sta63] J.D. Stasheff. Homotopy associativity of H-spaces, I, II. Trans. Am. Math. Soc., 108:275-292, 293312, 1963.

[Sta97] J.D. Stasheff. The pre-history of operads, in [Lod97], 9-14.

[Sta97a] J.D. Stasheff. From operads to physically inspired theories, in Lod97], 53-81. 
[Ton97] A. Tonks. Relating the associahedron and the permutohedron, in [Lod97], 33-36.

[Zie95] G.M. Ziegler, Lectures on Polytopes, Springer, Berlin, 1995.

This work is licensed under the Creative Commons Attribution-NoDerivs License. To view a copy of this license, visit http://creativecommons.org/licenses/by-nd/2.0/ or send a letter to Creative Commons, 171 Second St, Suite 300, San Francisco, CA 94105, USA, or Eisenacher Strasse 2, 10777 Berlin, Germany 